\title{
Anaplasma phagocytophilum Infection Subverts Carbohydrate Metabolic Pathways in the Tick Vector, Ixodes scapularis
}

\author{
Alejandro Cabezas-Cruz ${ }^{1,2 *}$, Pilar Alberdi ${ }^{3 \dagger}$, James J. Valdés ${ }^{1,4}$, Margarita Villar ${ }^{3}$ and \\ José de la Fuente ${ }^{3,5 *}$ \\ ${ }^{1}$ Institute of Parasitology, Biology Center, Czech Academy of Sciences, Ceské Budejovice, Czechia, ${ }^{2}$ Faculty of Science, \\ University of South Bohemia, Ceské Budejovice, Czechia, ${ }^{3} \mathrm{SaBio}$. Instituto de Investigación en Recursos Cinegéticos \\ (CSIC-UCLM-JCCM), Ciudad Real, Spain, ${ }^{4}$ Department of Virology, Veterinary Research Institute, Brno, Czechia, \\ ${ }^{5}$ Department of Veterinary Pathobiology, Center for Veterinary Health Sciences, Oklahoma State University, Stillwater, OK, \\ USA
}

The obligate intracellular pathogen, Anaplasma phagocytophilum, is the causative agent of human, equine, and canine granulocytic anaplasmosis and tick-borne fever (TBF) in ruminants. A. phagocytophilum has become an emerging tick-borne pathogen in the United States, Europe, Africa, and Asia, with increasing numbers of infected people

OPEN ACCESS

Edited by:

Robert Heinzen,

National Institute of Allergy and Infectious Diseases, USA

Reviewed by:

Yang Zhang,

University of Pennsy/vania, USA

Anders Omsland,

Washington State University, USA

*Correspondence:

Alejandro Cabezas-Cruz cabezasalejandrocruz@gmail.com José de la Fuente jose_delafuente@yahoo.com

${ }^{\dagger}$ These authors have contributed equally to this work.

Received: 25 November 2016 Accepted: 18 January 2017

Published: 07 February 2017

Citation:

Cabezas-Cruz A, Alberdi P, Valdés JJ, Villar M and de la Fuente J (2017) Anaplasma phagocytophilum Infection

Subverts Carbohydrate Metabolic Pathways in the Tick Vector, Ixodes scapularis.

Front. Cell. Infect. Microbiol. 7:23. doi: 10.3389/fcimb.2017.00023 and animals every year. It has been recognized that intracellular pathogens manipulate host cell metabolic pathways to increase infection and transmission in both vertebrate and invertebrate hosts. However, our current knowledge on how A. phagocytophilum affect these processes in the tick vector, Ixodes scapularis is limited. In this study, a genome-wide search for components of major carbohydrate metabolic pathways was performed in I. scapularis ticks for which the genome was recently published. The enzymes involved in the seven major carbohydrate metabolic pathways glycolysis, gluconeogenesis, pentose phosphate, tricarboxylic acid cycle (TCA), glyceroneogenesis, and mitochondrial oxidative phosphorylation and $\beta$-oxidation were identified. Then, the available transcriptomics and proteomics data was used to characterize the mRNA and protein levels of $I$. scapularis major carbohydrate metabolic pathway components in response to $A$. phagocytophilum infection of tick tissues and cultured cells. The results showed that major carbohydrate metabolic pathways are conserved in ticks. A. phagocytophilum infection inhibits gluconeogenesis and mitochondrial metabolism, but increases the expression of glycolytic genes. A model was proposed to explain how $A$. phagocytophilum could simultaneously control tick cell glucose metabolism and cytoskeleton organization, which may be achieved in part by up-regulating and stabilizing hypoxia inducible factor 1 alpha in a hypoxia-independent manner. The present work provides a more comprehensive view of the major carbohydrate metabolic pathways involved in the response to $A$. phagocytophilum infection in ticks, and provides the basis for further studies to develop novel strategies for the control of granulocytic anaplasmosis.

Keywords: proteomics, transcriptomics, glucose metabolism, Ixodes scapularis, Anaplasma phagocytophilum 


\section{INTRODUCTION}

Anaplasma phagocytophilum (Rickettsiales: Anaplasmataceae) is an obligate intracellular bacterium mainly transmitted by Ixodes spp. ticks. This emerging pathogen has been reported in the United States, Europe, Africa, and Asia (de la Fuente et al., 2008; Stuen et al., 2013; Kocan et al., 2015), causing human granulocytic anaplasmosis (HGA), equine and canine granulocytic anaplasmosis and tick-borne fever (TBF) of ruminants (de la Fuente et al., 2008; Stuen et al., 2013; Kocan et al., 2015).

The development of $A$. phagocytophilum is complex and coordinated with the tick feeding cycle. Infection and multiplication in ticks occurs first in midgut cells during blood feeding, and then subsequently in other tissues including hemocytes and salivary glands from where transmission occurs to susceptible hosts (Kocan et al., 2015). To establish infection, A. phagocytophilum affect mechanisms that appear to be common to ticks and vertebrate hosts (de la Fuente et al., 2016a). These mechanisms include but are not limited to remodeling of the cytoskeleton, inhibition of cell apoptosis, manipulation of the immune response, and modification of cell epigenetics and metabolism (de la Fuente et al., 2016a).

Recently, transcriptomics, proteomics and metabolomics analyses of infected I. scapularis ISE6 cells showed that A. phagocytophilum infection affects glucose metabolic pathways (Villar et al., 2015). These results suggested that $A$. phagocytophilum manipulate carbohydrate metabolism to facilitate infection and multiplication in tick cells. However, the mechanisms used by $A$. phagocytophilum for the manipulation of carbohydrate metabolic pathways have not been fully characterized.

To better characterize the mechanisms used by $A$. phagocytophilum to manipulate carbohydrate metabolic pathways during infection of tick cells, the dynamics of the carbohydrate metabolism was characterized in the tick vector, I. scapularis in response to pathogen infection. First, the composition of major carbohydrate metabolic pathways was annotated using the recently published genome of I. scapularis (Gulia-Nuss et al., 2016). Then, previously published transcriptomics and proteomics data (Ayllón et al., 2015; Villar et al., 2015) was used to characterize the mRNA and protein levels of carbohydrate metabolic pathway components in response to A. phagocytophilum infection of I. scapularis nymphs, female midguts and salivary glands, and ISE6 cultured tick cells. Finally, functional studies were conducted in ISE6 tick cells to provide additional support for the role of these components during pathogen infection. These results expanded our knowledge of the different pathways affected by A. phagocytophilum infection in ticks, and provided new potential targets for the development of therapeutic and prevention strategies for the control of granulocytic anaplasmosis and other tick-borne diseases.

\section{MATERIALS AND METHODS}

\section{Annotation of the Major Carbohydrate Metabolic Pathway Components in the I. scapularis Genome}

The I. scapularis genome (Gulia-Nuss et al., 2016) was searched with the specific names of genes encoding for enzymes involved in the major carbohydrate metabolic pathways, glycolysis, gluconeogenesis, pentose phosphate, tricarboxylic acid cycle (TCA), glyceroneogenesis, and mitochondrial oxidative phosphorylation and $\beta$-oxidation. When records were not obtained using specific enzyme names, then the I. scapularis genome was searched with the Blastp tool from the Basic Local Alignment Search Tool (BLAST) using the human ortholog as "query" (Altschul et al., 1990; Madden et al., 1996). The sequences with the lowest $E$-value were selected. The conserved domains of identified protein sequences were classified using the protein families database Pfam (Finn et al., 2014). The $I$. scapularis orthologs found in the genome were doublechecked by searching the Homo sapiens genome database using as queries the tick homologs identified in the previous step.

\section{Characterization of the I. scapularis mRNA and Protein Levels in Response to A. phagocytophilum Infection}

The quantitative transcriptomics and proteomics data for uninfected and A. phagocytophilum-infected I. scapularis nymphs, female midguts and salivary glands, and ISE6 cultured cells were obtained from previously published results (Ayllón et al., 2015; Villar et al., 2015) and deposited at the Dryad repository database, NCBI's Gene Expression Omnibus database and ProteomeXchange Consortium via the PRIDE partner repository with the dataset identifier PXD002181 and doi: 10.6019/PXD002181. For transcriptomics and proteomics analysis in I. scapularis nymphs, female midguts and salivary glands, the procedures were described in Ayllón et al. (2015). Briefly, nymphs and adult female I. scapularis were infected with $A$. phagocytophilum by feeding on a sheep inoculated intravenously with $\sim 1 \times 10^{7} \mathrm{~A}$. phagocytophilum (NY18 isolate)infected HL-60 cells (90-100\% infected cells). In this model, over $85 \%$ of ticks become infected with A. phagocytophilum in nymphs, midguts and salivary glands. Ticks (200 nymphs and 100 female adults) were removed from the sheep 7 days after infestation, held in the humidity chamber for 4 days and dissected for DNA, RNA, and protein extraction from whole internal tissues (nymphs) or midguts and salivary glands (adult females). Adult midguts and salivary glands were washed in PBS after collection to remove hemolymphs-related cells. Uninfected ticks were prepared in a similar way but feeding on an uninfected sheep. Two independent samples were collected and processed for each tick developmental stage and tissue. After RNA sequencing on an Illumina Hiseq 2000, TopHat was used to align the reads to the I. scapularis (assembly JCVI_ISG_i3_1.0; http://www.ncbi.nlm.nih.gov/ 
nuccore/NZ_ABJB000000000) reference genome. Raw counts per gene were estimated by the Python script HTSeq count [http://www-huber.embl.de/users/anders/HTSeq/] using the reference genome. The raw counts per gene were used by DEGseq to estimate differential expression at $P<0.05$. For peptide identification by liquid chromatography-tandem mass spectrometry (LC-MS/MS) using iTRAQ labeled peptides, all spectra were analyzed with Proteome Discoverer (version 1.4.0.29, Thermo Fisher Scientific) using a Uniprot database (http://www.uniprot.org) containing all sequences from Ixodida, Anaplasmataceae and Ruminantia. Peptide identification was validated using the probability ratio method and false discovery rate (FDR) was calculated using inverted databases and the refined method with an additional filtering for precursor mass tolerance of $12 \mathrm{ppm}$. Only peptides with a confidence of at least $95 \%$ were used to quantify the relative abundance of each peptide. Outliers at the scan and peptide levels and significant protein-abundance changes were detected from the $z$-values (the standardized variable used by the model that expresses the quantitative values in units of standard deviation) by using a FDR threshold of 5\%. Results were the mean of two replicates. For transcriptomics and proteomics analysis in tick cells, the I. scapularis embryo-derived tick cell line ISE6, provided by Ulrike Munderloh, University of Minnesota, USA, was cultured in L-15B300 medium (Munderloh et al., 1999), except that the osmotic pressure was lowered by the addition of one-fourth sterile water by volume. The ISE6 cells were first inoculated with A. phagocytophilum (human NY18 isolate; Asanovich et al., 1997)-infected HL-60 cells and maintained until infection was established and routinely passaged. Uninfected and infected cultures $(N=3$ independent cultures with $\sim 10^{7}$ cells each) were sampled at 7 days postinfection (dpi; percent infected cells 71-77\%; Ave $\pm S D$, $74 \pm 3$ ). Transcriptomics data was obtained as described above for nymphs and adult female ticks. Two biological replicates were used for each of uninfected and infected tick cells and genes differentially expressed in response to $A$. phagocytophilum infection were selected with $P \leq 0.05$. The proteomics analysis followed the same pipeline describe above in ticks and the MS/MS raw files generated with Xcalibur (version 2.1, Thermo Fisher Scientific) were searched against a compiled database containing all sequences from Ixodida and Anaplasmataceae (http://www.uniprot.org). Three biological replicates were used for each of uninfected and infected tick cells. For the quantitative analysis of tick proteins, after discarding Anaplasma proteins in infected cells, the total number of peptide-spectrum matches (PSMs) for each tick protein were normalized against the total number of PSMs in tick cells and compared between control and infected cells by Chi2-test $(P \leq 0.05)$. Although the percent of infected ticks and cultured cells was determined as described above, the bacterial load on these samples was not considered in the analysis.

The identified genes in the carbohydrate metabolic pathways were searched against the transcriptomics and proteomics data to characterize their mRNA and protein levels in response to $A$. phagocytophilum infection.

\section{Tertiary Structure Modeling and Optimization}

The tertiary structures of the partial protein sequences of I. scapularis hypoxia-inducible factor 1 alpha HIF- $1 \alpha$ (XP_002414889) and HIF-1 $\beta$ (XP_002416629) proteins, which are the DNA binding $\mathrm{N}$-terminus domains, were modeled using the Swiss-Model server (Biasini et al., 2014). The tertiary models were optimized using the Protein Preparation Wizard (Li et al., 2007) in the Schrödinger's Maestro software package. The Protein Preparation Wizard clusters at the highest degree of hydrogen bonding in equilibrium. Monte Carlo orientations are performed $(100,000)$ for each cluster. The optimized structure is based on electrostatic and geometric scoring functions. Any remaining steric clashes were eliminated by minimization of the entire system with the default settings in the Schrodinger's Maestro package. The ternary structure that includes the hypoxia response element (HRE) was constructed via superimposition by using the Swiss-Model server used the mouse HIF- $1 \alpha$, HIF-1 $\beta$, and the HRE (Dalei et al., 2015) as a template for both $I$. scapularis hypoxia-inducible protein sequences. As a final optimization step, the ternary structure (HIF-1 $\alpha / \mathrm{HIF}-1 \beta / \mathrm{HRE}$ ) was processed using the normal mode ready-made script in the Metropolis Monte Carlo-based Protein Energy Landscape Exploration (PELE) server (Borrelli et al., 2005). The PELE software implements an anisotropic network model (Atilgan et al., 2001) for perturbations of the alpha-carbon backbone causing structural conformational changes. The PELE server can be accessed at https://pele.bsc.es/.

\section{Immunofluorescence Assay in I. scapularis Midguts and Salivary Glands}

Female ticks fed on A. phagocytophilum-infected and uninfected sheep and fixed with $4 \%$ paraformaldehyde in $0.2 \mathrm{M}$ sodium cacodylate buffer were embedded in paraffin and used to prepare sections on glass slides as previously described (Ayllón et al., 2015). The paraffin was removed from the sections through two washes in xylene and the sections were hydrated by successive $5 \mathrm{~min}$ washes with a graded series of 100, 96, and $65 \%$ ethanol and finally with distilled water. Next, the slides were treated with Proteinase K (Dako, Barcelona, Spain) for 7 min, washed with $0,1 \%$ PBS-Tween 20 (Sigma-Aldrich, St. Louis, MI, USA) and blocked with $2 \%$ bovine serum albumin (BSA; Sigma-Aldrich) in PBS-Tween 20 during $1 \mathrm{~h}$ at room temperature. The slides were then incubated overnight at $4^{\circ} \mathrm{C}$ with mouse anti-Glyceraldehyde 3-phosphate dehydrogenase (GAPDH) monoclonal antibodies (ab50567; Abcam, Cambridge, UK) diluted 1:100 in 2\% BSA/PBS-Tween 20. Preimmune serum was used as control. After 3 washes with PBS-Tween 20, the slides were incubated for $1 \mathrm{~h}$ with rabbit anti-mouse IgG conjugated with FITC (Sigma-Aldrich) diluted 1:160 in 2\% BSA/PBS-Tween 20. Finally, after two washes with PBS the slides were mounted on ProLong Diamond Antifade Mountant with DAPI reagent (Thermo Scientific, Madrid, Spain). The sections were examined using a Zeiss LSM 800 with Airyscan (Carl Zeiss, Oberkochen, Germany). 


\section{Infected and Uninfected Cultured ISE6 Tick Cells}

The I. scapularis embryo-derived tick cell line ISE6 were cultured as described above, infected with A. phagocytophilum (human NY18 isolate; Asanovich et al., 1997) or mock-infected and maintained according to Munderloh et al. (1999).

\section{Pharmacological Studies in Cultured ISE6 Tick Cells}

A. phagocytophilum-infected ISE6 cells were left untreated or treated for 24 or $48 \mathrm{~h}$ with $5 \mathrm{mM}$ 2-Deoxy-D-Glucose (ab142242, Abcam) to inhibit glycolysis (Wang et al., 2011), $100 \mu \mathrm{M}$ LY294002 (ab120243, Abcam) to inhibit the phosphatidylinositol 3-kinase (PI3K; Sultana et al., 2010), 100 nM Chetomin (ab144222, Abcam) to inhibit the activity of HIF-1 $\alpha$ (Misra et al., 2012) or $0.5 \mathrm{mM}$ Deferoxamine mesylate (Sigma-Aldrich) to activate HIF-1 $\alpha$ (Choi et al., 2004). After treatment, cells were harvested for the preparation of whole cell lysates to determine HIF- $1 \alpha$ activity and for DNA extraction. Tick cell lysates were prepared by adding to cell pellets the RIPA lysis buffer (Thermo Scientific) supplemented with a protease inhibitor cocktail (cOmplete Mini, EDTA-free, Roche, Sigma-Aldrich). HIF- $1 \alpha$ activity was determined in the cell lysate supernatants with the HIF-1 alpha Transcription Factor Assay Kit (ab133104, Abcam) following manufacturer's recommendations. $A$. phagocytophilum DNA levels were characterized by msp4 real-time PCR normalizing against tick $16 S$ rDNA as described previously (Ayllón et al., 2013). Optical density values (O.D. $450 \mathrm{~nm}$ ) for HIF-1 $\alpha$ activity and normalized Ct values for $A$. phagocytophilum DNA levels were compared between treated and untreated control cells by Student's $t$-test with unequal variance $(P=0.05 ; N=4$ biological replicates).

\section{RESULTS}

\section{Major Carbohydrate Metabolic Pathways Described in Other Organisms Are Present in I. scapularis and Are Affected by A. phagocytophilum Infection}

Seven major pathways involved in carbohydrate metabolism were selected for characterization (Table 1). A total of 79 genes coding for the proteins involved in glycolysis, gluconeogenesis, pentose phosphate pathway (PPP), glyceroneogenesis, TCA, mitochondrial oxidative phosphorylation (OXPHOS) and $\beta$-oxidation were identified in the $I$. scapularis genome (Table 1). Based on these results, a model for glucose metabolism in ticks was proposed (Figure 1). At least in humans, pyruvate carboxylase (PC) catalyzes the irreversible carboxylation of pyruvate to form oxaloacetate, which is then transformed in phosphoenolpyruvate by the cytoplasmic enzyme phosphoenolpyruvate carboxykinase (PEPCK-C; Berg et al., 2002). However, the PC orthologue was not identified in the $I$. scapularis genome.

The carbohydrate metabolic response to A. phagocytophilum infection was then characterized using the quantitative transcriptomics and proteomics data generated from uninfected and A. phagocytophilum-infected I. scapularis ticks and ISE6 cultured cells (Ayllón et al., 2015; Villar et al., 2015). Most of the identified carbohydrate metabolism genes were differentially regulated in response to $A$. phagocytophilum infection in at least one of the analyzed tick tissues (Figure 2). Twenty-eight (35\%), 23 (29\%), $62(78 \%)$, and $64(81 \%)$ carbohydrate metabolism components were identified in both transcriptome and proteome of ISE6 cells, nymphs, adult female midguts, and salivary glands, respectively (Figure 2). Of these genes, 59 (75\%), 14 (18\%), $60(76 \%)$, and $41(52 \%)$ were up-regulated, while 20 (25\%), 65 (82\%), $16(20 \%)$, and 35 (44\%) were down-regulated in response to infection in ISE6 cells, nymphs, adult female midguts, and salivary glands, respectively (Figure 2). Many of the carbohydrate metabolism proteins were not identified by mass spectrometry (Figure 2), but the results showed similar differential regulation at the mRNA and protein levels for $50 \%$ of the identified proteins.

Glucose transporters, and mainly facilitative glucose transporters (GLUT) are an important component of the glycolytic pathway because they transport glucose from the extracellular space to the cellular cytoplasm, which makes the glucose accessible to hexokinase (HXK; Augustin, 2010; Li et al., 2015). We found 11 putative glucose transporters in the genome of I. scapularis, 2 members (sglt1 and sglt2) of the sodium-glucose linked transporter family (SGLT), and 9 members ( 2 isoforms of glut1, glut3, 4 isoforms of glut8, glut10, and glut12) of the GLUT family. In response to $A$. phagocytophilum infection, glut $1 B$, glut3, glut8C, glut10, glut12, and sglt1 genes were up-regulated in tick midguts, and the GLUT1A and SGLT2 proteins were overrepresented in infected midguts when compared to uninfected controls (Figure 3 ).

As in previous experiments (Villar et al., 2015), these results supported a role for carbohydrate metabolism during $A$. phagocytophilum infection in I. scapularis, and suggested tissuespecific differences in response to infection.

\section{A. phagocytophilum Infection Activates the Glycolysis Pathway, but Reduces Gluconeogenesis and the TCA Cycle in Tick Midguts}

Based on these results, the putative glucose metabolic pathways affected by $A$. phagocytophilum infection in different I. scapularis tissues and ISE6 cells were proposed (Figures 4, 5). The results showed that the genes involved in glycolysis were all up-regulated in tick midguts after $A$. phagocytophilum infection, except for phosphofructokinase $(p f k)$ and $h x k$ genes that did not change in response to infection (Figure 2). The up-regulation of glycolytic genes correlated with protein over-representation in tick midguts, except for the cofactor-independent phosphoglycerate mutase (ipgm) and pyruvate kinase ( $p k)$ that despite gene upregulation in infected midguts, protein was under-represented in response to infection (Figure 2). However, since iPGM catalyzes a reversible reaction, it is not a site of major regulatory mechanisms for the glycolytic pathway. In contrast, HXK and PFK proteins, which catalyze irreversible steps of glucose glycolysis and therefore constitute major regulatory 
TABLE 1 | Annotation of carbohydrate metabolic enzymes identified in the $I$. scapularis genome.

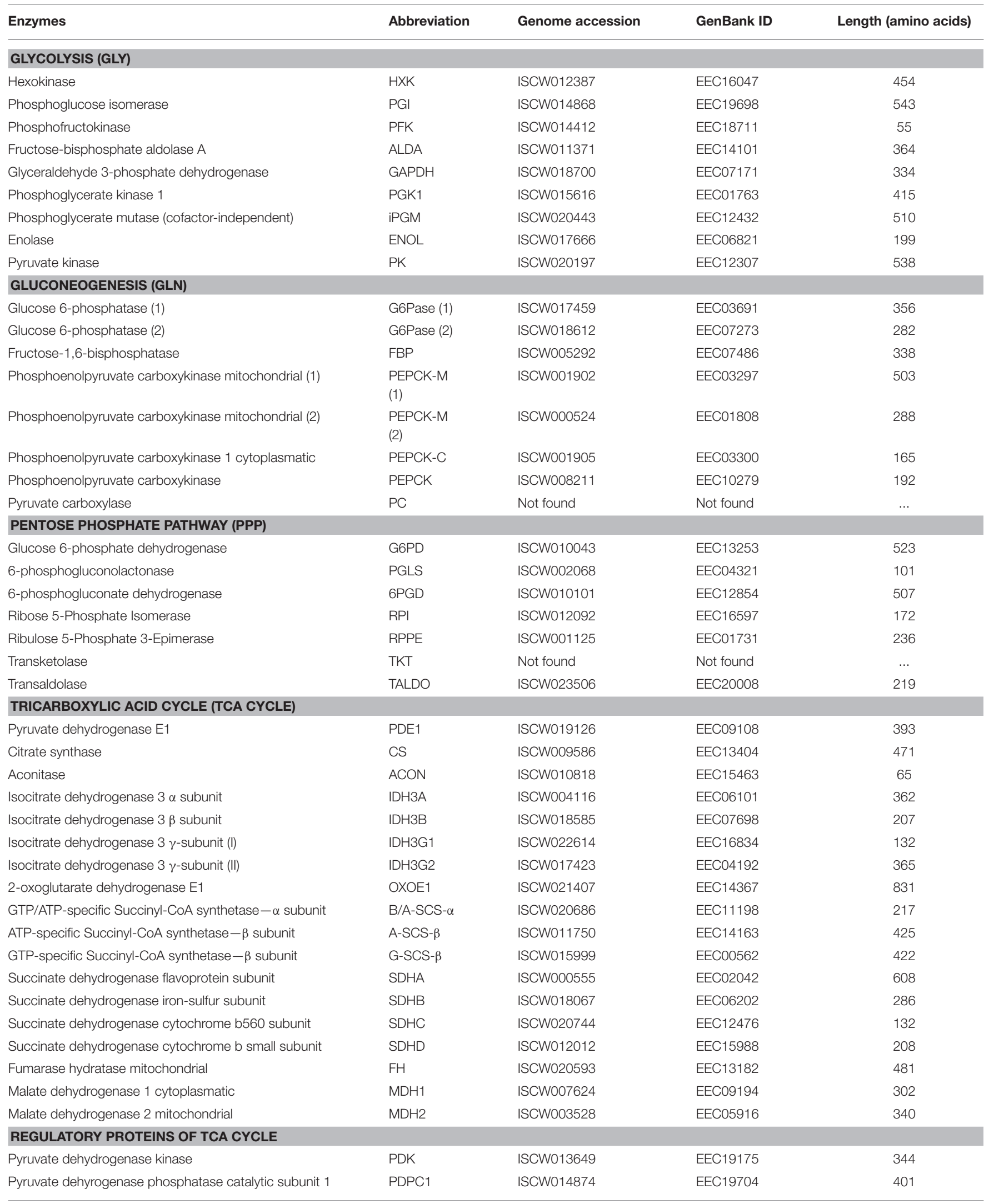


TABLE 1 | Continued

\begin{tabular}{|c|c|c|c|c|}
\hline Enzymes & Abbreviation & Genome accession & GenBank ID & Length (amino acids) \\
\hline \multicolumn{5}{|c|}{ OXIDATIVE PHOSPHORYLATION (OXPHOS) COMPLEX I (CORE SUBUNITS) } \\
\hline NADH dehydrogenase (ubiquinone) iron-sulfur protein 7 & NDUS7 & ISCW013042 & EEC17810 & 162 \\
\hline NADH dehydrogenase (ubiquinone) flavoprotein 2 & NDUV2 & ISCW014272 & EEC18480 & 244 \\
\hline NADH dehydrogenase (ubiquinone) iron-sulfur protein 3 & NDUS3 & ISCW012366 & EEC17298 & 268 \\
\hline NADH-ubiquinone oxidoreductase $75 \mathrm{kDa}$ subunit & NDUS1 & ISCW003299 & EEC03949 & 729 \\
\hline NADH-ubiquinone oxidoreductase chain 1 & ND1 & ISCW016073 & EEC02134 & 339 \\
\hline NADH-ubiquinone oxidoreductase chain 2 & ND2 & Not found & Not found & $\ldots$ \\
\hline NADH-ubiquinone oxidoreductase chain 3 & ND3 & Not found & Not found & $\ldots$ \\
\hline NADH-ubiquinone oxidoreductase chain 4 & ND4 & Not found & Not found & $\ldots$ \\
\hline \multicolumn{5}{|l|}{ OXPHOS COMPLEX II } \\
\hline Succinate dehydrogenase flavoprotein subunit & SDHA & ISCW000555 & EEC02042 & 608 \\
\hline Succinate dehydrogenase iron-sulfur subunit & SDHB & ISCW018067 & EEC06202 & 286 \\
\hline Succinate dehydrogenase cytochrome b560 subunit & $\mathrm{SDHC}$ & ISCW020744 & EEC12476 & 132 \\
\hline Succinate dehydrogenase cytochrome b small subunit & SDHD & ISCW012012 & EEC15988 & 208 \\
\hline \multicolumn{5}{|l|}{ OXPHOS COMPLEX III } \\
\hline Cytochrome b-c1 complex subunit 1 & QCR1 & Not found & Not found & $\ldots$ \\
\hline Cytochrome b-c1 complex subunit 2 & QCR2 & Not found & Not found & $\ldots$ \\
\hline Cytochrome b & CYTB & ISCW016730 & EEC02834 & 385 \\
\hline Cytochrome c1 & CYTC1 & ISCW016731 & EEC02835 & 251 \\
\hline Cytochrome b-c1 complex subunit Rieske & RIESKE & ISCW014071 & EEC19414 & 229 \\
\hline Cytochrome c oxidase subunit 1 & $\operatorname{cox} 1$ & Not found & ADO64507 & 276 \\
\hline Cytochrome c oxidase subunit 2 & $\operatorname{cox} 2$ & ISCW016021 & EEC02091 & 244 \\
\hline Cytochrome c oxidase subunit 3 & $\operatorname{cox} 3$ & ISCW020757 & EEC11360 & 278 \\
\hline Cytochrome c oxidase subunit 4 & COX4 & Not found & AAY66918 & 179 \\
\hline Cytochrome c oxidase subunit 5A & COX5A & ISCW008682 & EEC12772 & 153 \\
\hline Cytochrome c oxidase subunit 5B & COX5B & ISCW019526 & EEC10693 & 124 \\
\hline Cytochrome $\mathrm{c}$ oxidase subunit $6 \mathrm{~A}$ & COX6A & ISCW019235 & EEC08541 & 111 \\
\hline Cytochrome c oxidase subunit 6B & COX6B & ISCW015139 & EEC20473 & 82 \\
\hline Cytochrome c oxidase subunit $6 \mathrm{C}$ & COX6C & ISCW016559 & EEC02407 & 76 \\
\hline Cytochrome c oxidase subunit 7 & COX7 & Not found & Not found & $\ldots$ \\
\hline Cytochrome c oxidase subunit 8 & COX8 & ISCW024928 & EEC20083 & 68 \\
\hline \multicolumn{5}{|c|}{ OXPHOS COMPLEX V (ATP SYNTHASE: F1-ATP SYNTHASE STRUCTURE/FO-ATP SYNTHASE STRUCTURE) } \\
\hline ATP synthase subunit $\alpha 1$ (F1) & ATPSA1 & ISCW011988 & EEC16574 & 555 \\
\hline ATP synthase subunit $\alpha 2$ (F1) & ATPSA2 & ISCW019217 & EEC08626 & 355 \\
\hline ATP synthase subunit $\beta$ (F1) & ATPSB & ISCW012509 & EEC17118 & 563 \\
\hline ATP synthase subunit $\gamma(\mathrm{F} 1)$ & ATPSG & ISCW023477 & EEC19169 & 314 \\
\hline ATP synthase subunit $\delta(F 1)$ & ATPSD & ISCW018418 & EEC06183 & 168 \\
\hline
\end{tabular}


TABLE 1 | Continued

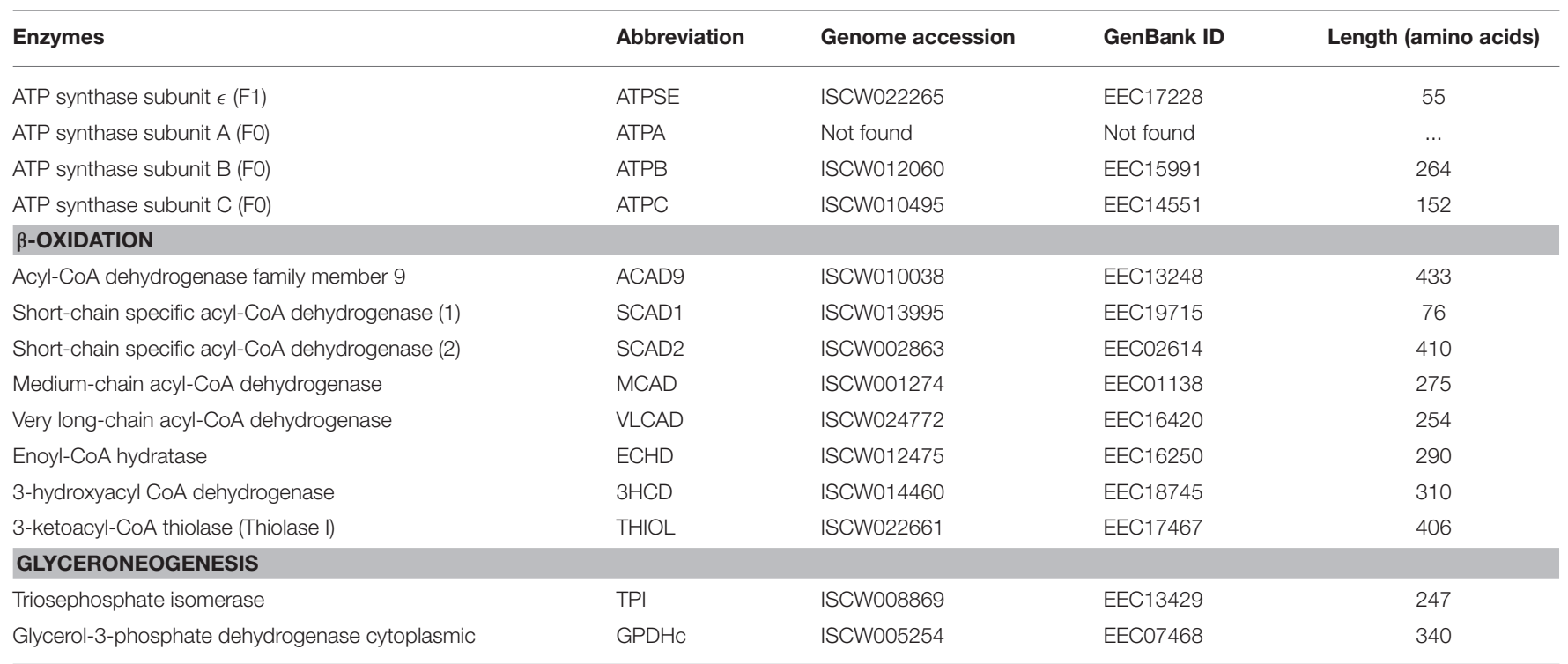

steps of this pathway, were over-represented and did not change, respectively in response to infection of tick midguts (Figure 4). The expression of the p53 target TP53-inducible glycolysis and apoptosis regulator (tigar, ISCW020485), which was recently shown to inhibit glycolysis (Bensaad et al., 2006), was down-regulated in tick nymphs, midguts, salivary glands, and ISE6 cells. The over-representation of all glycolytic enzymes correlated with up-regulation of their respective genes, suggesting transcriptional regulation. These data suggested that A. phagocytophilum infection activates glucose uptake and degradation in $I$. scapularis midguts. In agreement with an increase in glucose catabolism, the levels of glucose were significantly reduced $(0.51 \pm 0.06$ vs. $0.31 \pm 0.05, p<0.005)$ in infected ISE6 cells when compared to uninfected controls (Villar et al., 2015).

PK catalyzes the final irreversible step of glycolysis, the transfer of a phosphate group from phosphoenolpyruvate to ADP, yielding one molecule of pyruvate and one molecule of adenosine 5'-triphosphate (ATP; Li et al., 2015). This gene was up-regulated, but protein was under-represented in response to infection in tick midguts (Figures 2, 4). Due to the regulatory role of $\mathrm{PK}$, low levels of this enzyme may hamper the flux of pyruvate toward the mitochondrial matrix, decreasing the activity of TCA cycle. PK generates pyruvate, which is transported to the mitochondrial matrix by mitochondrial pyruvate carriers 1 and 2 (MPC1 and MPC2). The homologs for $m p c 1$ and $m p c 2$ genes were identified in I. scapularis and were up-regulated in tick midguts, but down-regulated in salivary glands in response to $A$. phagocytophilum infection (Figure 3). In the mitochondrial matrix, pyruvate is decarboxylated by pyruvate dehydrogenase E1 (PDE1; Berg et al., 2002). The decarboxylation of pyruvate is the first step of a series of enzymatic reactions that transform pyruvate into acetyl coenzyme A (acetyl-Coa), which is oxidized to $\mathrm{CO}_{2}$ and water in the TCA cycle (Berg et al., 2002). PDE1 is an important regulator of the TCA cycle (Berg et al., 2002). Pyruvate dehydrogenase kinase 1 (PDK1) inhibits, while pyruvate dehydrogenase phosphatase catalytic subunit 1 (PDPC1) activates PDE1 (Berg et al., 2002). We found that in the midguts of infected ticks, PDE1 was under-represented and the genes $p d k 1$ and $p d p c 1$ were up-regulated and down-regulated, respectively in response to infection (Figure 2). This result strongly suggested that A. phagocytophilum infection inhibited the TCA cycle in $I$. scapularis midguts. In agreement with this suggestion, several TCA cycle enzymes such as citrate synthase (CS), aconitase $(\mathrm{ACON})$, isocitrate dehydrogenase [subunits $\alpha$ (IDH3A) and $\gamma$ (IDH3G1)], 2-oxoglutarate dehydrogenase E1 (OXOE1), succinate dehydrogenase (flavoprotein subunit (SDHA), and iron-sulfur subunit (SDHB) were under-represented in midguts from infected ticks when compared to uninfected controls (Figures 2, 4).

The NADH generated by the TCA cycle is fed into the OXPHOS pathway to produce ATP (Li et al., 2015). The mRNA and protein levels of components of the OXPHOS complex I-IV present in I. scapularis were examined. Higher mRNA and protein levels were found for most of the respiratory components in infected tick midguts when compared to uninfected controls (Figure 2). This result suggested that ticks might have a regulatory mechanism to maintain the levels of ATP in response to the inhibition of the TCA cycle.

Finally, we found that although most genes were upregulated, all proteins of gluconeogenesis (synthesis of glucose from non-carbohydrate precursors) were under-represented in tick midguts, except for fructose-1,6-bisphosphatase (FBP; Figure 2). FBP catalyzes an irreversible reaction in the gluconeogenesis, the conversion of fructose 1,6-bisphosphate into fructose 6-phosphate, and therefore it is a regulatory step of this anabolic pathway. However, the enzyme glucose 


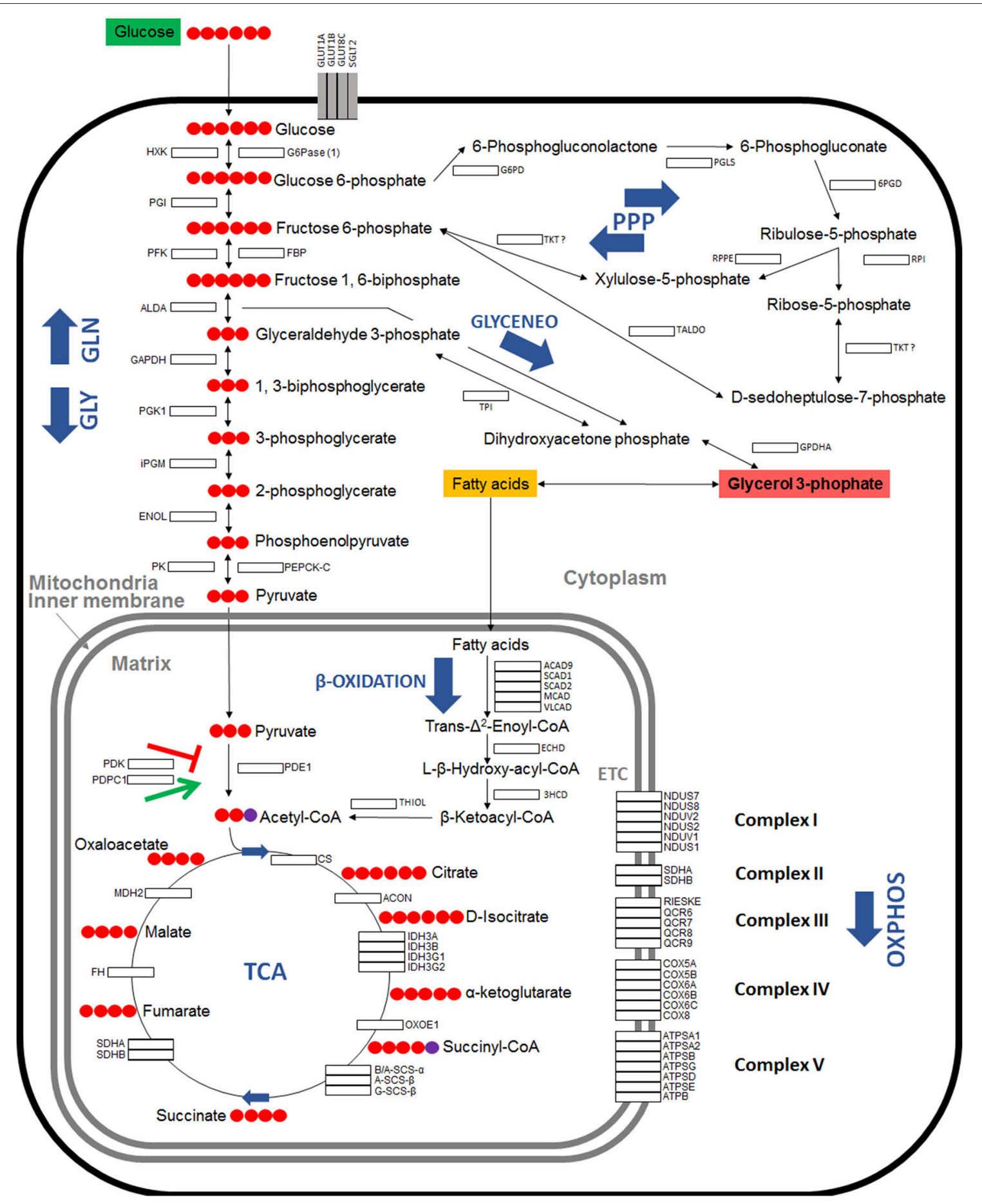

FIGURE 1 | Model of carbohydrate metabolism in I. scapularis. The main enzymes involved in Glycolysis (GLY), Gluconeogenesis (GLN), Pentose phosphate pathway (PPP), Tricarboxylic acid cycle (TCA), Oxidative phosphorylation (OXPHOS) complex I to V, $\beta$-Oxidation and Glyceroneogenesis (GLYCENEO) and present in the genome of I. scapularis (Table 1) are shown. The names and number of carbon molecules (Red circles) of the metabolic intermediates of these metabolic pathways are also shown. The names of the enzymes were abbreviated for the different pathways as GLY: Hexokinase (HXK), Phosphoglucose isomerase (PGI), Phosphofructokinase (PFK), Fructose-bisphosphate aldolase A (ALDA), Glyceraldehyde 3-phosphate dehydrogenase (GAPDH), Phosphoglycerate kinase 1 (PGK1), Phosphoglycerate mutase (cofactor-independent) (iPGM), Enolase (ENOL), Pyruvate kinase (PK); GLN: Glucose 6-phosphatase (1) (G6Pase (1)), Fructose-1,6-bisphosphatase (FBP), Phosphoenolpyruvate carboxykinase mitochondrial (1) (PEPCK-M (1)), Phosphoenolpyruvate carboxykinase mitochondrial (2) (PEPCK-M (2)), Phosphoenolpyruvate carboxykinase 1 cytoplasmatic (PEPCK-C), Pyruvate carboxylase (PC); PPP: Glucose 6-phosphate dehydrogenase (G6PD), 6-phosphogluconolactonase (PGLS), 6-phosphogluconate dehydrogenase (6PGD), Ribose 5-Phosphate Isomerase (RPI), Ribulose 5-Phosphate 3-Epimerase (RPPE), Transketolase (TKT), Transaldolase (TALDO); $\beta$-Oxidation: Acyl-CoA dehydrogenase family member 9 (ACAD9), Short-chain specific acyl-CoA dehydrogenase 
FIGURE 1 | Continued

(1) (SCAD1), Short-chain specific acyl-CoA dehydrogenase (2) (SCAD2), Medium-chain acyl-CoA dehydrogenase (MCAD), Very long-chain acyl-CoA dehydrogenase (VLCAD), Enoyl-CoA hydratase (ECHD), 3-hydroxyacyl CoA dehydrogenase (3HCD), 3-ketoacyl-CoA thiolase (Thiolase I) (THIOL); GLYCENEO: Triosephosphate isomerase (TPI), Glycerol-3-phosphate dehydrogenase cytoplasmic (GPDHc); OXPHOS: Complex I: NADH dehydrogenases (NDUS7, NDUS8, NDUV2, NDUS3, NDUS2 and NDUV1), NADH-ubiquinone oxidoreductases (NDUS1, ND1 and ND5); Complex II: Succinate dehydrogenases (SDHA, SDHB, SDHC, SDHD); Complex III: Cytochrome b and b-c1 complexe subunits (QCR1, QCR2, CYTB, RIESKE, QCR6, QCR7, QCR8, QCR9, QCR10, QCR11), Cytochrome c1 (CYTC1); Complex IV: Cytochrome c oxidase subunits (COX1, COX2, COX3, COX5A, COX5B, COX6A, COX6B, COX6C, COX8); Complex V: ATP synthase subunits (ATPSA1, ATPSA2, ATPSB, ATPSG, ATPSD, ATPSE, ATPB, ATPC).

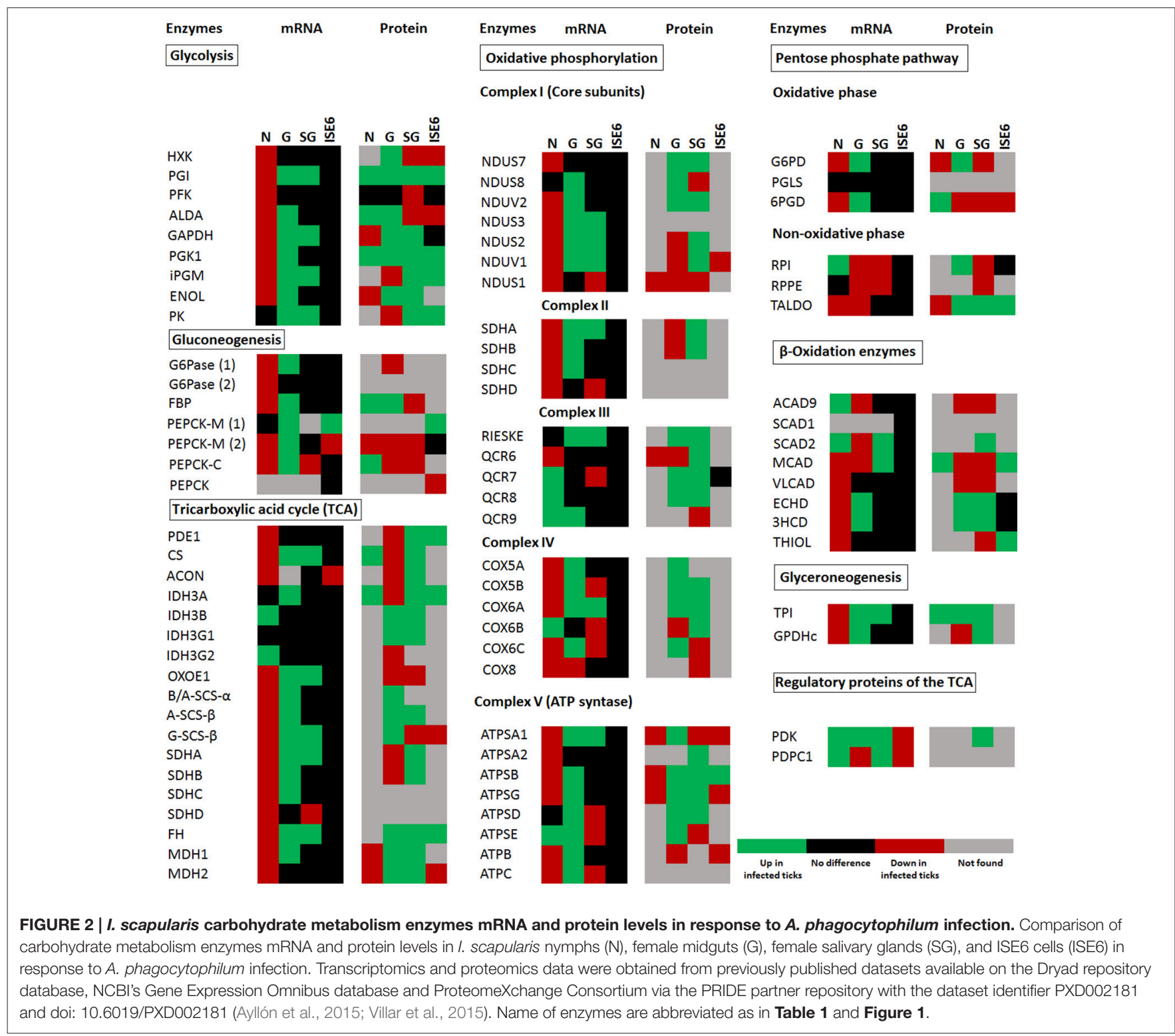

6-phosphatase that catalyzes the last step of gluconeogenesis, the transformation of glucose 6-phosphate into free glucose was under-represented. These results suggested that midgut cells in A. phagocytophilum-infected ticks tend to keep glucose 6-phosphate within the cells for other metabolic processes (e.g., glycogen synthesis).

\section{HIF-1 Components Are Up-Regulated in I. scapularis Infected with}

\section{A. phagocytophilum}

HIF-1 is a heterodimeric transcription factor consisting of a constitutively expressed $\beta$-subunit (HIF-1 $\beta$ ) and an oxygenregulated $\alpha$-subunit (HIF-1 $\alpha$; Pagé et al., 2002; Déry et al., 2005; 


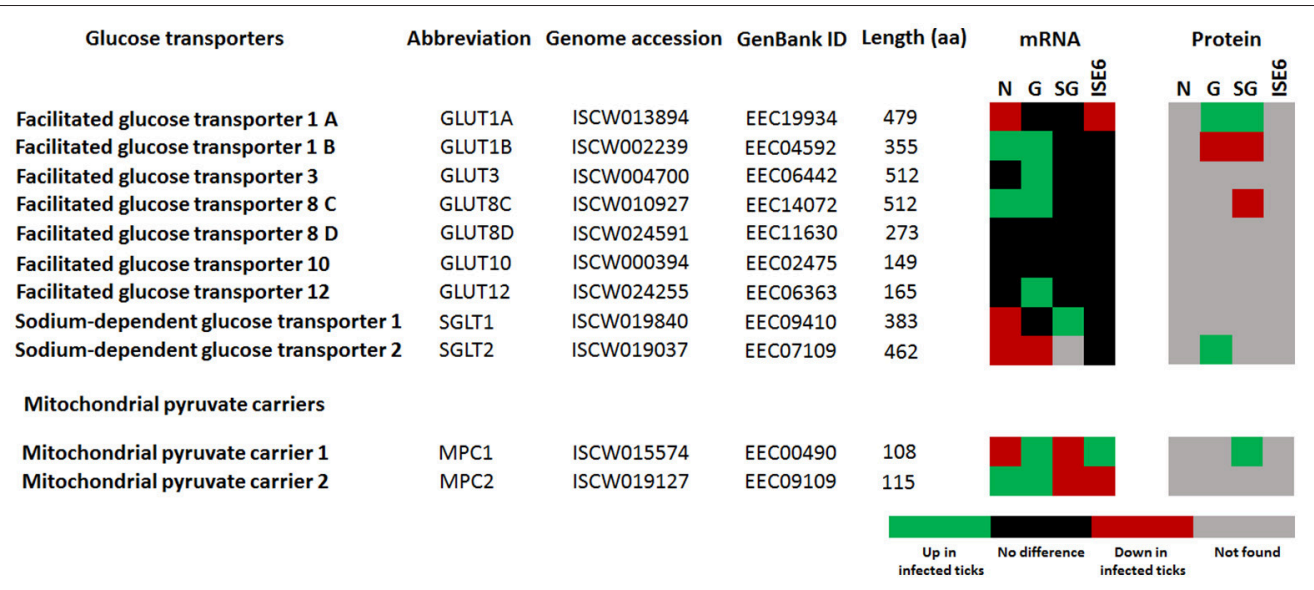

FIGURE 3 | I. scapularis glucose transporters mRNA and protein levels in response to $\boldsymbol{A}$. phagocytophilum infection. Comparison of glucose transporters mRNA and protein levels in I. scapularis nymphs (N), female midguts (G), female salivary glands (SG) and ISE6 cells (ISE6) in response to A. phagocytophilum infection. Transcriptomics and proteomics data were obtained from previously published datasets available on the Dryad repository database, NCBl' Gene Expression Omnibus database and ProteomeXchange Consortium via the PRIDE partner repository with the dataset identifier PXD002181 and doi: 10.6019/PXD002181 (Ayllón et al., 2015; Villar et al., 2015).

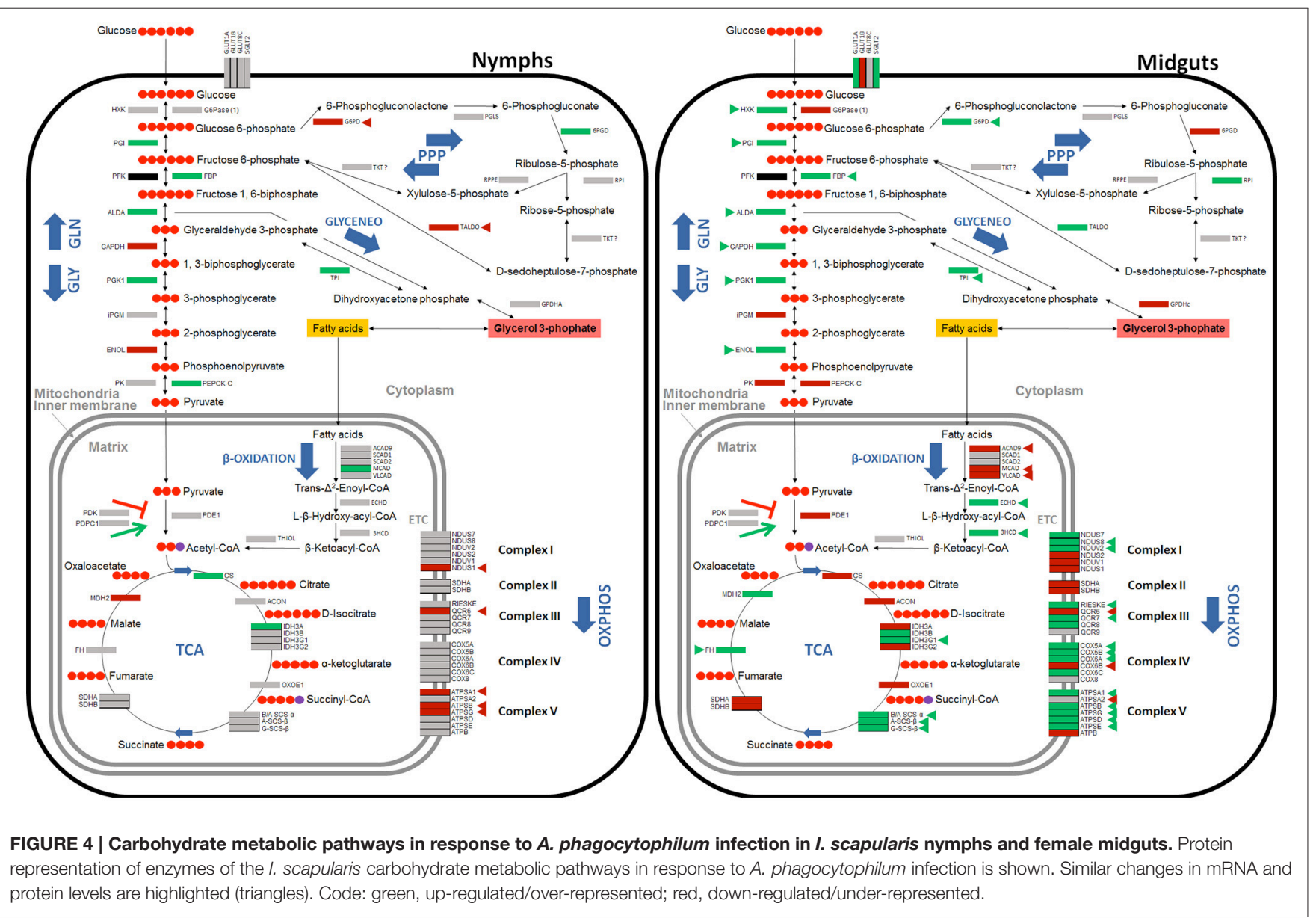

Ziello et al., 2007; Badeaux and Shi, 2013). The gene encoding for HIF-1 $\alpha$ (ISCW023657), which is a major transcriptional activator of glycolytic genes ( $\mathrm{Hu}$ et al., 2006), was significantly up-regulated in tick midguts, but not in nymphs, salivary glands or ISE6 cells in response to A. phagocytophilum infection (Ayllón et al., 2015; Villar et al., 2015). The expression of the gene 

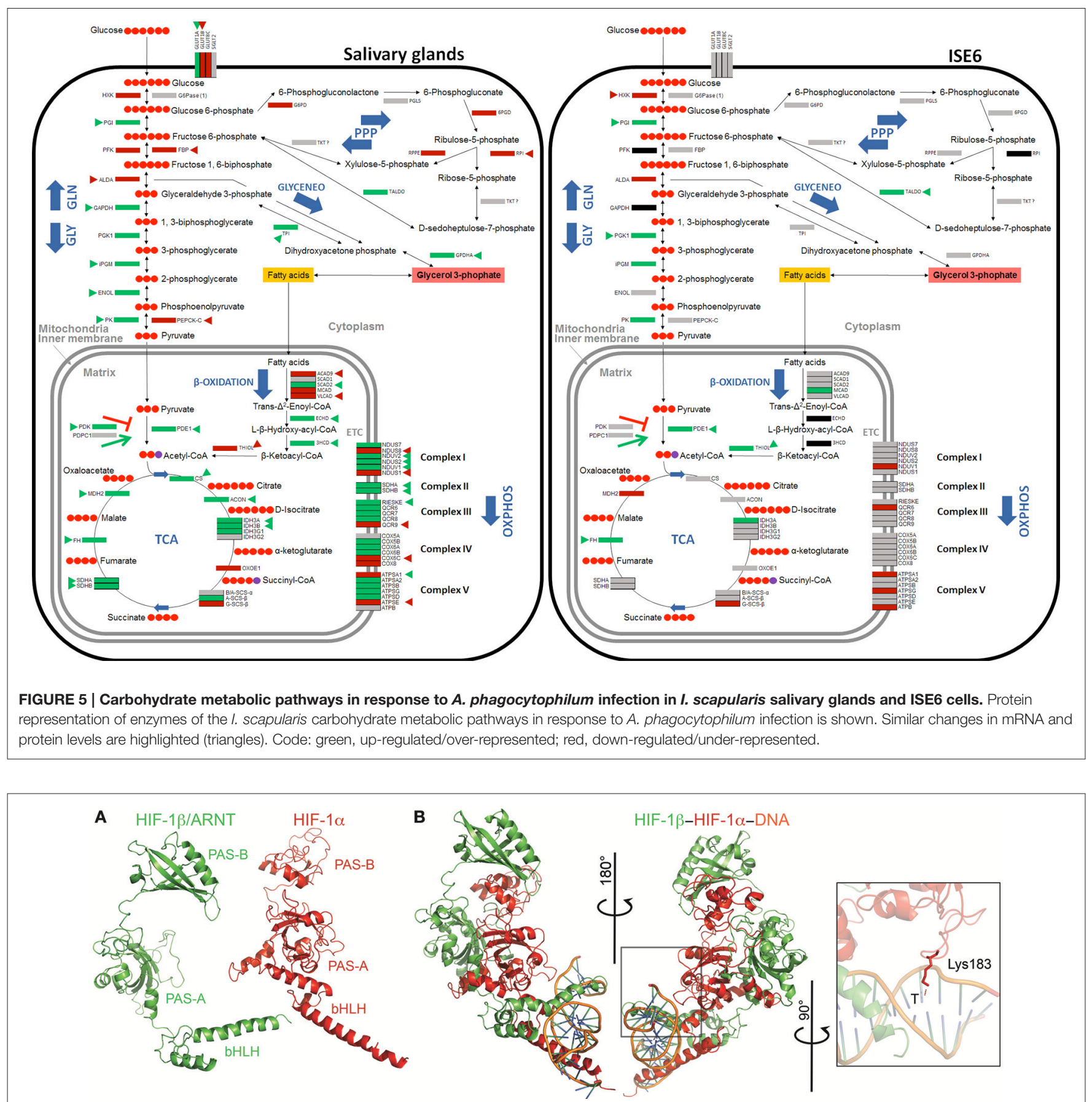

FIGURE 6 | Modeling of the HIF-1 $\alpha$-HIF-1 $\beta$-HRE complex. (A) HIF-1 $\alpha$ (red) and HIF-1 $\beta$ (green) monomers with their respective domains labeled. (B) Ternary complex of both monomers plus the hypoxia response element (HRE; orange). The inset shows the conserved Lys residue that interacts with the thymine (T) of HRE.

encoding for the HIF-1 $\beta$ (ISCW023999), which is a molecular partner of HIF-1 $\alpha$, was up-regulated in midguts from infected ticks, but was down-regulated in nymphs and did not change in salivary glands and ISE6 cells in response to infection (Ayllón et al., 2015; Villar et al., 2015).

Both I. scapularis HIF-1 $\alpha$ and HIF-1 $\beta$ (a.k.a, aryl hydrocarbon receptor nuclear translocator or ARNT) possess the conserved basic helix-loop-helix (bHLH) domain and two PAS domains (Figures 6A,B). The bHLH domain of HIF- $1 \alpha$ and HIF-1 $\beta$ were highly conserved with identical interacting residues as those previously identified (Dalei et al., 2015). The PAS-B domain of HIF-1 $\alpha$ was disordered due to its low conservation and the presence of small insertions and deletions when compared to the mouse HIF- $1 \alpha$ (Dalei et al., 2015). The PAS-B of HIF-1 $\alpha$ 
interacts with both the PAS domain of HIF-1 $\beta$, thereby making it difficult to determine interacting residues. One key feature of HIF- $1 \alpha$ is the presence of two residues lysine (Lys) and glycine (Gly), which interact with the nucleotides of hypoxia response element (HRE; Dalei et al., 2015). The I. scapularis HIF-1 $\alpha$ had the conserved Lys, but showed an asparagine (Asn) to Gly substitution (Figure 6).

\section{The PI3K/Akt Pathway Is Present in}

\section{I. scapularis and Regulated in Response to} A. phagocytophilum Infection

It has been demonstrated that a number of non-hypoxic stimuli are highly capable of inducing the expression of hif-1 (Pagé et al., 2002; Déry et al., 2005). The PI3K pathway and its downstream effectors, mTOR and p70S6 kinase (p70S6K), may induce a hypoxia-independent increase in HIF-1 $\alpha$ levels (Déry et al., 2005). In addition, diacylglycerol (DAG)-sensitive protein kinases $\mathrm{C}(\mathrm{PKC})$ were shown to up-regulate hif- $1 \alpha$ gene expression in a hypoxia-independent manner (Pagé et al., 2002; Déry et al., 2005). Infection with A. phagocytophilum activates PI3K in I. scapularis (Sultana et al., 2010). Therefore, we characterized the mRNA and protein levels of tick PI3K components in response to A. phagocytophilum infection.
Most PI3K-mTOR pathway components found in other organisms (Pilot-Storck et al., 2010) were identified in the $I$. scapularis genome (Table 2). The tick PI3K components were differentially regulated in response to A. phagocytophilum infection (Figure 7). The $p 70 S 6 \mathrm{~K}$ and PI3K components ( $p 85 \alpha$ and $p 110 \alpha)$ were up-regulated in tick midguts and salivary glands in response to infection. Additionally, p70S6K and p85 $\alpha$ proteins were over-represented in midguts from infected ticks when compared to uninfected controls (Figure 7). Oxygenindependent activation of hif- $1 \alpha$ may involve PI3K components, p70S6K and mTOR (Déry et al., 2005). However, we did not find an mTOR ortholog in the I. scapularis genome. DAG-sensitive PKCs were also suggested to play a role in oxygen-independent activation of hif-1 $\alpha$ (Pagé et al., 2002). Protein PKC $\alpha$ was found to be over-represented in tick midguts and salivary glands, while the $P K C \varepsilon$ gene was up-regulated in tick midguts in response to infection.

\section{Functional Studies Support a Role for Carbohydrate Metabolism during A. phagocytophilum Infection of Tick Cells} Functional studies were focused on glycolysis by targeting the process at different levels (Figures 8A,B).

TABLE 2 | Annotation of PI3K-mTOR pathway components identified in the l. scapularis genome.

\begin{tabular}{|c|c|c|c|c|}
\hline Proteins & Abbreviation & Genome accession & GenBank ID & Length (amino acids) \\
\hline RAC-alpha serine/threonine-protein kinase & AKT2 & ISCW018284 & EEC06033 & 371 \\
\hline Phosphatidylinositol 4,5-bisphosphate 3-kinase catalytic subunit $\alpha$ & $\mathrm{p} 110 \alpha$ & ISCW014229 & EEC18977 & 617 \\
\hline Phosphatidylinositol 4,5-bisphosphate 3-kinase catalytic subunit $\beta$ & $\mathrm{p} 110 \beta$ & ISCW010469 & EEC14480 & 491 \\
\hline Phosphatidylinositol-4-phosphate 3-kinase C2 domain-containing $\alpha$ (1) & $\mathrm{PI} 3 \mathrm{~K}-\mathrm{C} 2 \alpha 1$ & ISCW022811 & EEC17358 & 1638 \\
\hline Phosphatidylinositol-4-phosphate 3-kinase C2 domain-containing $\alpha$ (2) & $\mathrm{PI} 3 \mathrm{~K}-\mathrm{C} 2 \alpha 2$ & ISCW004026 & EEC06376 & 1142 \\
\hline Phosphatidylinositol 3-kinase regulatory subunit $\alpha$ & $\mathrm{p} 85 \alpha$ & ISCW011707 & EEC13970 & 427 \\
\hline 3- phosphoinositide-dependent protein kinase 1 & PDK1 & ISCW021115 & EEC15400 & 521 \\
\hline Phosphatidylinositol 3,4,5-trisphosphate 5-phosphatase 1 & SHIP1 & ISCW000015 & EEC00572 & 535 \\
\hline Phosphatidylinositol 3,4,5-trisphosphate 3-phosphatase PTEN & PTEN & ISCW021211 & EEC15363 & 363 \\
\hline Arf-GAP with GTPase, ANK repeat and PH domain-containing protein & PIKE & ISCW014072 & EEC18950 & 558 \\
\hline Glycogen synthase kinase $3 \beta$ (2) & GSK3ß2 & ISCW009451 & EEC11886 & 588 \\
\hline Hamartin & TSC1 & ISCW023319 & EEC19233 & 874 \\
\hline Tuberin & TSC2 & ISCW012320 & EEC16560 & 429 \\
\hline Serine/threonine kinase 11 (1) & LKB1/STK11A & ISCW024898 & EEC19357 & 170 \\
\hline Serine/threonine kinase 11 (2) & LKB1/STK11B & ISCW015294 & EEC20092 & 293 \\
\hline AMP-activated protein kinase $\alpha$ & $\mathrm{AMPK} \alpha$ & ISCW020637 & EEC13103 & 510 \\
\hline AMP-activated protein kinase $\beta$ & AMPK $\beta$ & ISCW021036 & EEC15614 & 183 \\
\hline AMP-activated protein kinase $\gamma$ & $\mathrm{AMPK} \gamma$ & ISCW010164 & EEC13342 & 154 \\
\hline Ras homolog enriched in brain & RHEB & ISCW018020 & EEC05182 & 182 \\
\hline Regulatory-associated protein of mTOR & RAPTOR & ISCW018753 & EEC07385 & 995 \\
\hline G protein $\beta$ subunit-like & $\mathrm{G} \beta \mathrm{L}$ & ISCW002765 & EEC03813 & 324 \\
\hline Rapamycin-insensitive companion of mTOR & RICTOR & ISCW018420 & EEC06185 & 987 \\
\hline Phosphatidylinositol 3-kinase catalytic subunit type 3 & Vps34 & ISCW008679 & EEC12769 & 864 \\
\hline Serine/threonine-protein kinase VPS15 & Vps15 & ISCW017661 & EEC06819 & 1351 \\
\hline Pleckstrin homology domain-containing, family $\mathrm{O}$ member 1 & CKIP1 & ISCW021605 & EEC15223 & 359 \\
\hline Casein kinase 2 subunit $\alpha$ & $\mathrm{CK} 2 \alpha$ & ISCW007259 & EEC10594 & 436 \\
\hline Casein kinase 2 subunit $\beta$ & CK2 $2 \beta$ & ISCW014459 & EEC18744 & 222 \\
\hline Ribosomal protein S6 kinase beta-1 (p70s6 kinase ) & S6K1/p70s6K & ISCW017390 & EEC03348 & 344 \\
\hline
\end{tabular}


A. phagocytophilum-infected ISE6 cells were left untreated or treated for 24 or $48 \mathrm{~h}$ with 2-Deoxy-D-Glucose to inhibit glycolysis (Wang et al., 2011), LY294002 to inhibit the PI3K (Sultana et al., 2010), Chetomin to inhibit the activity of HIF-1 $\alpha$ (Misra et al., 2012) or Deferoxamine mesylate to activate HIF-1 $\alpha$ (Figures 8A,B). Both HIF-1 $\alpha$ (Ayllón et al., 2015; Villar et al., 2015), which is a major transcriptional activator of glycolytic genes (Hu et al., 2006), and most PI3K components (Figure 7) were affected in response to $A$. phagocytophilum infection. The results showed that LY294002 was the only compound active in ISE6 tick cells (Figure 8A). The lack of effect of chetomin and Deferoxamine mesylate on tick HIF- $1 \alpha$ activity may be due to structural differences between tick and mammalian HIF-1 $\alpha$. The treatment of tick cells with LY294002 for $48 \mathrm{~h}$ resulted in lower HIF- $1 \alpha$ activity when compared to untreated control cells (Figure 8A). Treatment with LY294002 has been shown in $I$. scapularis and other organisms to inhibit the PI3K pathway (Sultana et al., 2010), which is involved in the induction of HIF-1 $\alpha$ to activate the glycolysis (Déry et al., 2005). Therefore, the results obtained in tick cells supported a role for PI3K in HIF- $1 \alpha$ induction in tick cells. The decrease in $A$. phagocytophilum infection after LY294002 treatment for 24 and $48 \mathrm{~h}$ (Figure 8B) provided additional support to these results. As expected from the lack of effect on HIF- $1 \alpha$ activity after incubation of tick cells with the other compounds, treatment did not affect $A$. phagocytophilum infection in these cells. Additionally, the immunofluorescence assay of GAPDH, which was over-represented in I. scapularis midguts and salivary glands in response to $A$. phagocytophilum infection (Figures 4, 5), corroborated the results of the proteomics analysis in tick salivary glands (Figure $\mathbf{8 C}$ ).

\section{DISCUSSION}

Recently, Villar et al. (2015) showed modifications in tick cell glucose metabolism during A. phagocytophilum infection. The results evidenced that infection affected the glucose metabolic pathway in tick cells through phosphoenolpyruvate carboxykinase (PEPCK) inhibition leading to decreased gluconeogenesis, which also results in the inhibition of cell apoptosis that increases pathogen infection of tick cells (Villar et al., 2015). Furthermore, these results provided evidence that other carbohydrate metabolic pathways are also affected by pathogen infection. Therefore, the objective of this study was the characterization of the dynamics of major carbohydrate pathways during A. phagocytophilum infection of I. scapularis.

Cellular glycolysis converts glucose to pyruvate, which enters the mitochondria where it is converted into acetyl-CoA, and is metabolized via the TCA cycle yielding reducing equivalents that are used for OXPHOS to generate ATP (Eisenreich et al., 2013 , 2015). TCA cycle can be also fueled by the acetylCoA produced via degradation of fatty acids after $\beta$-Oxidation (Eisenreich et al., 2013). Alternatively, glucose can be used to produce Ribose 5-phosphate, a precursor for the synthesis of nucleotides through the PPP (Berg et al., 2002; Figure 1). In addition, glyceroneogenesis uses glycerol 3-phophate, obtained

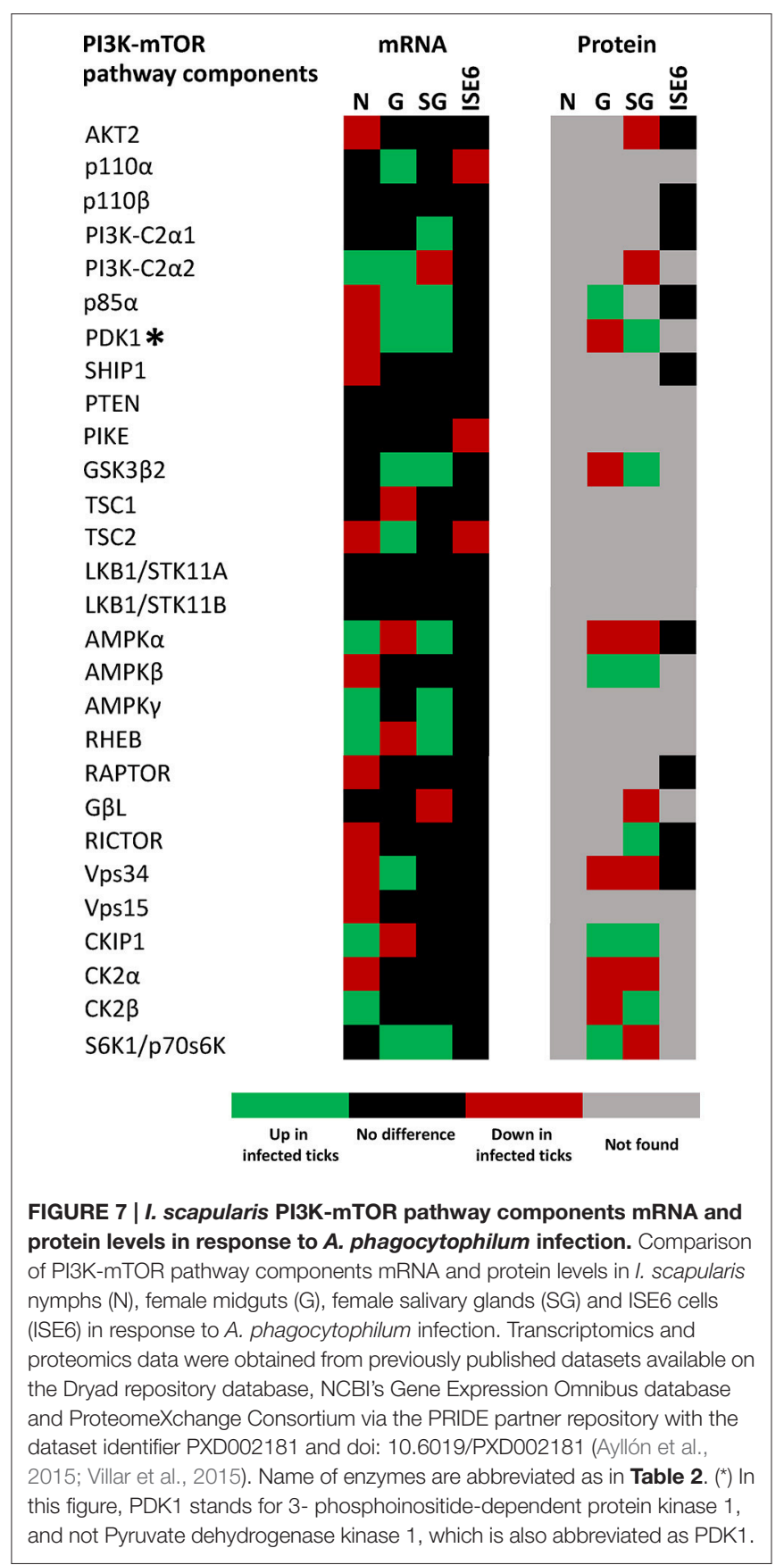

from the reduction of dihydroxyacetone phosphate (glycolytic intermediate) to synthetize fatty acids, and therefore is a major link between carbohydrate metabolism and lipid metabolism (Berg et al., 2002; Figure 1).

In this study, orthologs for 79 components of major carbohydrate metabolic pathways were identified in $I$. scapularis, and their role was characterized in response to A. phagocytophilum infection. The analysis was focused on seven pathways including glycolysis, gluconeogenesis, PPP, glyceroneogenesis, $\beta$-Oxidation, TCA cycle, and OXPHOS. The results showed that genes involved in glycolysis 

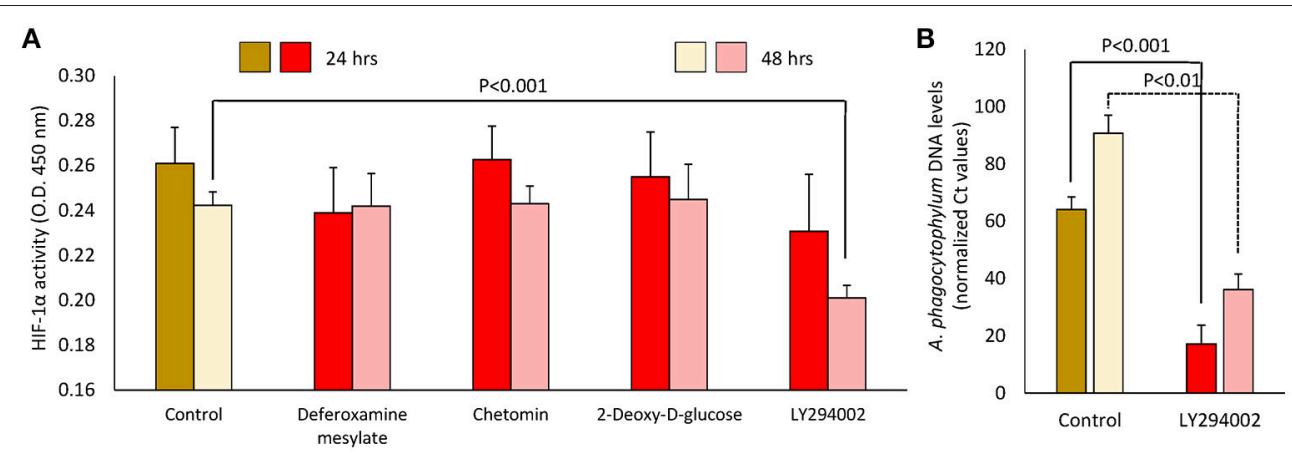

C
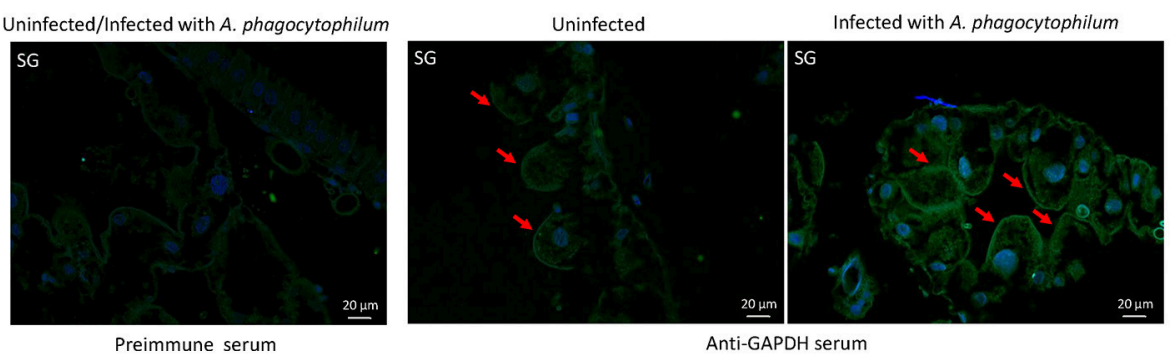

FIGURE 8 | Functional analysis supports a role for glycolysis during $\boldsymbol{A}$. phagocytophilum infection of tick cells. (A) A. phagocytophilum-infected ISE6 cells were left untreated (Control) or treated for 24 or 48 h with 2-Deoxy-D-Glucose to inhibit glycolysis, LY294002 to inhibit the PI3K, Chetomin to inhibit the activity of $\mathrm{HIF}-1 \alpha$ or Deferoxamine mesylate to activate HIF-1 $\alpha$. HIF-1 $\alpha$ activity was determined in cell lysates, and O.D. $450 \mathrm{~nm}$ values were shown as average + SD and compared between treated and untreated control cells by Student's $t$-test with unequal variance $(P=0.05 ; N=4$ biological replicates). (B) $A$. phagocytophilum DNA levels were characterized in LY294002-treated and untreated control cells by msp4 real-time PCR normalizing against tick 16S rDNA. Normalized Ct values were compared between treated and untreated control cells by Student's $t$-test with unequal variance $(P<0.01 ; N=4$ biological replicates). (C) Representative images of imunofluorescence analysis of uninfected and A. phagocytophilum-infected adult female $I$. scapularis salivary gland acini (SG). Tick tissues were stained with preimmune control serum or mouse anti-GAPDH monoclonal antibodies (green, FITC) or DAPI (blue) and superimposed (FITC+DAPI). Bars, 20 $\mu \mathrm{m}$. Red arrows illustrate the positive staining for GAPDH in tick SG.

were up-regulated in $I$. scapularis ticks infected with $A$. phagocytophilum. In contrast, PEPCK, which is the enzyme that catalyzes the first step of gluconeogenesis (after this step the molecules are "committed" to the pathway and will ultimately end up in the pathway's final product) was underrepresented (Figure 2). However, in addition to low levels of PEPCK, low TCA cycle activity might be also necessary to control gluconeogenesis (Burgess et al., 2007). Interestingly, we found that several enzymes and intermediates such as succinate of the TCA cycle are also under-represented in infected tick ISE6 cells and midguts (Figures 4, 5; Villar et al., 2015). These results strongly suggested that upon A. phagocytophilum infection, glycolysis is enhanced, TCA cycle inhibited and in agreement with our previous results (Villar et al., 2015), gluconeogenesis is inhibited. These findings supported the idea that $A$. phagocytophilum infection might be an energydemanding process for the tick cells and that the pathogen may benefit from glycolytic intermediates.

Intracellular bacteria trigger diverse host metabolic responses (Eisenreich et al., 2013, 2015). Numerous transcriptome studies have identified some of these metabolic responses as unspecific (triggered by extracellular/intracellular and pathogenic/nonpathogenic bacteria) and are therefore called "core host responses" (Boldrick et al., 2002). These "core host responses" are modulated in different ways by virulence mechanisms of different bacteria species (Boldrick et al., 2002). For example, in vivo studies in mice showed that the facultative intracellular pathogen Mycobacterium tuberculosis induces a reduction in the levels of glucose and the TCA cycle intermediates oxaloacetate and fumarate, but also an increase in lactate and succinate concentration (Shin et al., 2011; Somashekar et al., 2011). In Listeria monocytogenes, Lecuit et al. (2007) found that the transcription of most glycolytic genes was enhanced after infection in mice, particularly $h x k$ II. The upregulation of glycolytic genes was linked to enhanced hif- $1 \alpha$ expression and downregulation of the gluconeogenic gene $f b p$ (Lecuit et al., 2007). The above examples illustrate commonalities among the host metabolic responses to $M$. tuberculosis, L. monocytogenes and A. phagocytophilum infection.

Based on the results of this study, a model was proposed in which A. phagocytophilum induces transcriptional activation of hif- $1 \alpha$ through PI3K (p85 $\alpha, \mathrm{p} 110 \alpha$, and p70S6K) and PKC to activate the glycolytic pathway and inhibit the TCA cycle in infected ticks (Figure 9). This model was partially supported by functional studies using a PI3K inhibitor to monitor the effect on HIF-1 $\alpha$ activity and pathogen infection (Figures 8A,B). In normoxia, HIF- $1 \alpha$ is rapidly degraded. Therefore, A. phagocytophilum may have additional mechanisms to stabilize HIF-1 $\alpha$ at normal oxygen concentrations. The increase in the levels of heat shock proteins HSP70 and HSP90 


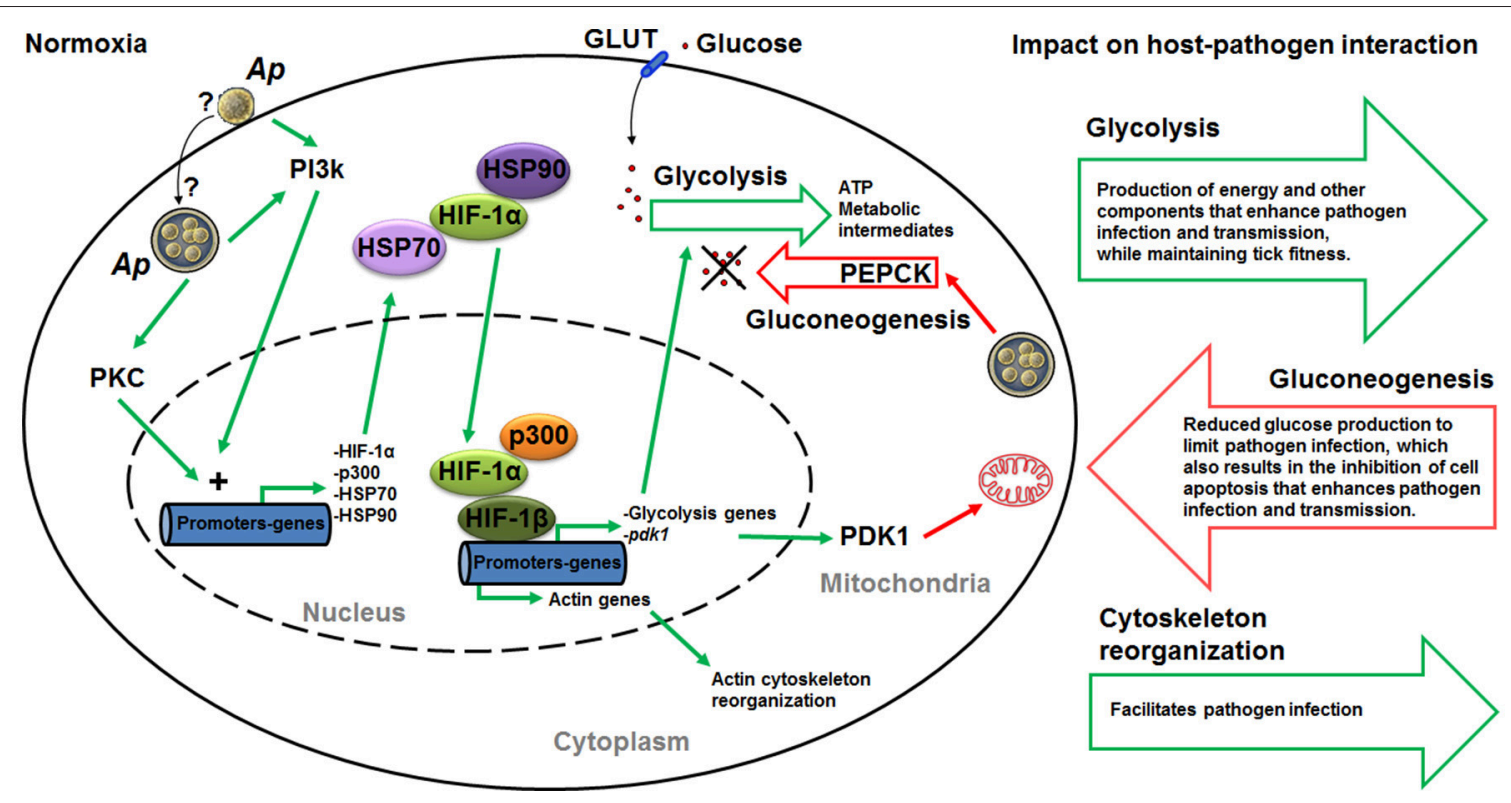

FIGURE 9 | Mechanistic model of carbohydrate metabolism manipulation by $\boldsymbol{A}$. phagocytophilum. We propose a mechanism by which $A$. phagocytophilum enhances glycolysis through HIF activation under normoxic conditions. Upon contact with the host membrane or once inside the parasitophorous vacuole, A. phagocytophilum induces the activation of PI3K and PKC that ultimately induce the expression of heat shock proteins HSP70 and HSP90, the acetyltransferase p300 and hif- $1 \alpha$. In normoxia, HSP70 and HSP90 stabilize HIF-1 $\alpha$, making possible the recruitment of p300 and HIF- $1 \alpha$ to form a complex with HIF- $1 \beta$ in the nucleus. This complex activates the expression of HIF-1 $\alpha$ target genes, which include glycolytic genes and pdk 1 . This mechanism will result in the increase in glycolysis and inhibition of gluconeogenesis. Furthermore, HIF activation can also leads to actin cytoskeleton reorganization. Therefore, by activating the HIF system A. phagocytophilum may regulate at the same time the tick cell carbohydrate metabolism and cytoskeleton organization. Green and red lines indicate induction/activation and inhibition, respectively. Question marks indicate that we do not know whether A. phagocytophilum induces the activation of PI3K and PKC upon contact with the host membrane or once inside the parasitophorous vacuole. Ap, A. phagocytophilum; PI3K, phosphatidylinositol 3-kinase pathway; diacylglycerol (DAG)-sensitive protein kinases C (PKC); HIF, hypoxia-inducible factor; PDK1, pyruvate dehydrogenase kinase 1; GLUT, facilitative glucose transporters; PEPCK, phosphoenolpyruvate carboxykinase.

may contribute to the stabilization of HIF- $1 \alpha$ in normoxia (Zhou et al., 2004). Previously, we showed that HSP70 and HSP90 were over-represented in A. phagocytophilum-infected ISE6 tick cells when compared to uninfected cells (Villar et al., 2015). Furthermore, mobilization of p300 at HIF target genes may be an additional requirement of HIF-mediated transcriptional activation (Badeaux and Shi, 2013). In agreement with this model, recently we showed that A. phagocytophilum induces the production of p300 to inhibit cell apoptosis and increase bacterial multiplication in tick cells (Cabezas-Cruz et al., 2016). By inhibiting the TCA cycle in host cells, A. phagocytophilum may inhibit the entrance of glutamine and glutamate to the TCA cycle via $\alpha$-ketoglutarate, which is one of the intermediates in the TCA cycle. This process may increase the cytoplasmic concentration of glutamine and glutamate that can then be used by $A$. phagocytophilum. The TCA cycle of A. phagocytophilum is incomplete (because one gene for isocitrate dehydrogenase is missing from the genome) and requires the exogenous acquisition of glutamine and glutamate (Huang et al., 2007), which the pathogen may obtain from the host cell cytoplasm. A. phagocytophilum has the enzyme that converts glutamine to glutamate and glutamate can be then transformed to $\alpha$-ketoglutarate that may fuel the bacterial TCA cycle.
Interestingly, it was recently shown that inhibition of prolyl hydroxylases, which are involved in the regulation of HIF-1 $\alpha$ activity, induces HIF- $1 \alpha$-dependent cytoskeletal remodeling in endothelial cells (Weidemann et al., 2013). Therefore, it is possible that A. phagocytophilum activates HIF$1 \alpha$ through PI3K to regulate simultaneously the carbohydrate metabolism and cytoskeleton organization to facilitate infection and multiplication in tick cells (Figure 9).

The comparison of metabolic pathways between selected Anaplasmataceae showed that Rickettsia prowazekii, Ehrlichia chaffeensis, Neorickettsia sennetsu, Wolbachia pipientis, and A. phagocytophilum might not be able to actively carry out glycolysis. Therefore, glycolytic metabolic intermediates produced by host cells may be necessary for the development of these bacteria. Only those glycolysis enzymes necessary to produce glyceraldehyde-3-phosphate and dihydroxyacetone phosphate from phosphoenolpyruvate are present in the genome of these Rickettsia spp. (Dunning Hotopp et al., 2006). Therefore, phosphoenolpyruvate may be one of the glycolytic intermediates that $A$. phagocytophilum hijacks from the host cell cytoplasm. Additionally, glycerol-3-phosphate, which is a product of glyceroneogenesis, was proposed as another metabolite taken from the host by Rickettsia spp. (Dunning Hotopp et al., 2006). In agreement with this proposed model, 
the glyceroneogenesis enzymes triosephosphate isomerase (TPI) and glycerol-3-phosphate dehydrogenase cytoplasmic (GPDHc) were over-represented in infected tick nymphs, midguts and/or salivary glands when compared to uninfected controls.

The failure to identify I. scapularis orthologs for some genes may be due to the absence of these pathway components in ticks or the fact that only $\sim 57 \%$ of the genome have been sequenced and assembled for this species (de la Fuente et al., 2016b; Gulia-Nuss et al., 2016). These results were similar to those obtained before for other genes and proteins in response to A. phagocytophilum infection, showing tissue-specific differences in the response to pathogen infection (Ayllón et al., 2015). As previously discussed, these results suggested that differences between mRNA and protein levels could be due to delay between mRNA and protein accumulation which requires sampling at different time points and/or the role for post-transcriptional and post-translational modifications in the tick tissue-specific response to A. phagocytophilum infection (Ayllón et al., 2015; Villar et al., 2015; Cabezas-Cruz et al., 2016).

\section{CONCLUSIONS}

These results support that major carbohydrate metabolic pathways are conserved in ticks. A. phagocytophilum infection has a major impact on the regulation of carbohydrate metabolic pathways in tick cells. As a result of the studies reported here, a mechanism was proposed by which this pathogen might induce the expression and stabilization of HIF- $1 \alpha$ to increase glycolysis, suppress TCA cycle to reduce gluconeogenesis, and regulate cytoskeleton organization (Figure 9). This may be achieved by a coordinated action of PI3K/PKC pathway, for induction of hif- $1 \alpha$ expression, and HSP70/90 and p300, for HIF-1 $\alpha$

\section{REFERENCES}

Altschul, S. F., Gish, W., Miller, W., Myers, E. W., and Lipman, D. J. (1990). Basic local alignment search tool. J. Mol. Biol. 215, 403-410. doi: 10.1016/S0022-2836(05)80360-2

Asanovich, K. M., Bakken, J. S., Madigan, J. E., Aguero-Rosenfeld, M., Wormser, G. P., and Dumler, J. S. (1997). Antigenic diversity of granulocytic Ehrlichia isolates from humans in Wisconsin and New York and a horse in California. J. Infect. Dis. 176, 1029-1034. doi: 10.1086/516529

Atilgan, A. R., Durell, S. R., Jernigan, R. L., Demirel, M. C., Keskin, O., and Bahar, I. (2001). Anisotropy of fluctuation dynamics of proteins with an elastic network model. Biophys. J. 80, 505-515. doi: 10.1016/S0006-3495(01)76033-X

Augustin, R. (2010). The protein family of glucose transport facilitators: it's not only about glucose after all. IUBMB Life 62, 315-333. doi: 10.1002/iub.315

Ayllón, N., Villar, M., Busby, A. T., Kocan, K. M., Blouin, E. F., BonzónKulichenko, E., et al. (2013). Anaplasma phagocytophilum inhibits apoptosis and promotes cytoskeleton rearrangement for infection of tick cells. Infect. Immun. 81, 2415-2425. doi: 10.1128/IAI.00194-13

Ayllón, N., Villar, M., Galindo, R. C., Kocan, K. M., Šíma, R., López, J. A., et al. (2015). Systems biology of tissue-specific response to Anaplasma phagocytophilum reveals differentiated apoptosis in the tick vector Ixodes scapularis. PLoS Genet. 11:e1005120. doi: 10.1371/journal.pgen.1005120

Badeaux, A. I., and Shi, Y. (2013). Emerging roles for chromatin as a signal integration and storage platform. Nat. Rev. Mol. Cell. Biol. 14, 211-224. doi: $10.1038 / \mathrm{nrm} 3545$ stabilization. The increase in glycolysis results in the production of energy and other components that enhance pathogen infection and transmission while preserving tick fitness. The reduction in gluconeogenesis may be a cell response to limit pathogen infection, but also results in the inhibition of cell apoptosis to enhance pathogen infection and transmission. These mechanisms provided additional support for the co-evolution of tick-pathogen interactions that can produce both conflict and cooperation between them (de la Fuente et al., 2016c). The identification of these mechanisms provided additional evidences to support that $A$. phagocytophilum uses similar strategies to infect vertebrate hosts and ticks (de la Fuente et al., 2016a), therefore suggesting the possibility of developing strategies for a more effective control of $A$. phagocytophilum and its associated diseases by targeting similar mechanisms in both vertebrate hosts and tick vectors.

\section{AUTHOR CONTRIBUTIONS}

$\mathrm{AC}$ and JF conceived the study. PA performed the experiments. AC, PA, JV, MV, and JF performed data analyses. AC and JF wrote the paper, and other co-authors made additional suggestions and approved the manuscript.

\section{FUNDING}

This research was supported by the Ministerio de Economia y Competitividad (Spain) grant BFU2016-79892-P and the European Union (EU) Seventh Framework Programme (FP7) ANTIGONE project number 278976. MV was supported by the Research Plan of the University of Castilla-La Mancha (UCLM), Spain.

Bensaad, K., Tsuruta, A., Selak, M. A., Vidal, M. N., Nakano, K., Bartrons, R., et al. (2006). TIGAR, a p53-inducible regulator of glycolysis and apoptosis. Cell 126, 107-120. doi: 10.1016/j.cell.2006.05.036

Berg, J. M., Tymoczko, J. L., and Stryer, L. (2002). Biochemistry, 5th Edn. New York, NY: W H Freeman Press.

Biasini, M., Bienert, S., Waterhouse, A., Arnold, K., Studer, G., Schmidt, T., et al. (2014). SWISS-MODEL: modelling protein tertiary and quaternary structure using evolutionary information. Nucleic. Acids. Res. 42, W252-W258. doi: 10.1093/nar/gku340

Boldrick, J. C., Alizadeh, A. A., Diehn, M., Dudoit, S., Liu, C. L., Belcher, C. E., et al. (2002). Stereotyped and specific gene expression programs in human innate immune responses to bacteria. Proc. Natl. Acad. Sci. U.S.A. 99, 972-977. doi: $10.1073 /$ pnas. 231625398

Borrelli, K. W., Vitalis, A., Alcantara, R., and Guallar, V. (2005). PELE: protein energy landscape exploration. A novel Monte Carlo based technique. J. Chem. Theor. Comput. 1, 1304-1311. doi: 10.1021/ct0501811

Burgess, S. C., He, T., Yan, Z., Lindner, J., Sherry, A. D., Malloy, C. R., et al. (2007). Cytosolic phosphoenolpyruvate carboxykinase does not solely control the rate of hepatic gluconeogenesis in the intact mouse liver. Cell Metab. 5, 313-320. doi: 10.1016/j.cmet.2007.03.004

Cabezas-Cruz, A., Alberdi, P., Ayllón, N., Valdés, J. J., Pierce, R., Villar, M., et al. (2016). Anaplasma phagocytophilum increases the levels of histone modifying enzymes to inhibit cell apoptosis and facilitate pathogen infection in the tick vector Ixodes scapularis. Epigenetics 11, 303-319. doi: 10.1080/15592294.2016.1163460 
Choi, E. Y., Kim, E. C., Oh, H. M., Kim, S., Lee, H. J., Cho, E. Y., et al. (2004). Iron chelator triggers inflammatory signals in human intestinal epithelial cells: involvement of p38 and extracellular signal-regulated kinase signaling pathways. J. Immunol. 172, 7069-7077. doi: 10.4049/jimmunol.172.11.7069

Dalei, W., Nalini, P., Jingping, L., Youngchang, K., and Fraydoon, R. (2015). Structural integration in hypoxia-inducible factors. Nature 524, 303-308. doi: $10.1038 /$ nature 14883

de la Fuente, J., Estrada-Peña, A., Cabezas-Cruz, A., and Kocan, K. M. (2016a). Anaplasma phagocytophilum uses common strategies for infection of ticks and vertebrate hosts. Trends Microbiol. 24, 173-180. doi: 10.1016/j.tim.2015.12.001

de la Fuente, J., Estrada-Pena, A., Venzal, J. M., Kocan, K. M., and Sonenshine, D. E. (2008). Overview: ticks as vectors of pathogens that cause disease in humans and animals. Front. Biosci. 13, 6938-6946. doi: 10.2741/3200

de la Fuente, J., Villar, M., Cabezas-Cruz, A., Estrada-Peña, A., Ayllón, N., and Alberdi, P. (2016c). Tick-host-pathogen interactions: conflict and cooperation. PLoS Pathog. 12:e1005488. doi: 10.1371/journal.ppat.1005488

de la Fuente, J., Waterhouse, R. M., Sonenshine, D. E., Roe, R. M., Ribeiro, J. M., and Sattelle, D. B. (2016b). Tick genome assembled: new opportunities for research on tick-host-pathogen interactions. Front. Cell. Infect. Microbiol. 6:103. doi: 10.3389/fcimb.2016.00103

Déry, M. A., Michaud, M. D., and Richard, D. E. (2005). Hypoxia-inducible factor 1: regulation by hypoxic and non-hypoxic activators. Int. J. Biochem. Cell. Biol. 37, 535-540. doi: 10.1016/j.biocel.2004.08.012

Dunning Hotopp, J. C., Lin, M., Madupu, R., Crabtree, J., Angiuoli, S. V., Eisen, J. A., et al. (2006). Comparative genomics of emerging human ehrlichiosis agents. PLoS Genet. 2:e21. doi: 10.1371/journal.pgen.0020021

Eisenreich, W., Heesemann, J., Rudel, T., and Goebel, W. (2013). Metabolic host responses to infection by intracellular bacterial pathogens. Front. Cell. Infect. Microbiol. 3:24. doi: 10.3389/fcimb.2013.00024

Eisenreich, W., Heesemann, J., Rudel, T., and Goebel, W. (2015). Metabolic adaptations of intracellullar bacterial pathogens and their mammalian host cells during infection ("Pathometabolism"). Microbiol. Spectr. 3, 27-58. doi: 10.1128/microbiolspec.MBP-0002-2014

Finn, R. D., Bateman, A., Clements, J., Coggill, P., Eberhardt, R. Y., Eddy, S. R., et al. (2014). Pfam: the protein families database. Nucleic Acids Res. 42, D222-D230. doi: $10.1093 /$ nar/gkt1223

Gulia-Nuss, M., Nuss, A. B., Meyer, J. M., Sonenshine, D. E., Roe, R. M., Waterhouse, R. M., et al. (2016). Genomic insights into the Ixodes scapularis tick vector of Lyme disease. Nat. Commun. 7:10507. doi: $10.1038 /$ ncomms10507

Hu, C. J., Iyer, S., Sataur, A., Covello, K. L., Chodosh, L. A., and Simon, M. C. (2006). Differential regulation of the transcriptional activities of hypoxiainducible factor $1 \alpha$ (HIF-1 $\alpha)$ and HIF-2 $\alpha$ in stem cells. Mol. Cell. Biol. 26, 3514-3526. doi: 10.1128/MCB.26.9.3514-3526.2006

Huang, H., Wang, X., Kikuchi, T., Kumagai, Y., and Rikihisa, Y. (2007). Porin activity of Anaplasma phagocytophilum outer membrane fraction and purified P44. J. Bacteriol. 189, 1998-2006. doi: 10.1128/JB.01548-06

Kocan, K. M., de la Fuente, J., and Cabezas-Cruz, A. (2015). The genus Anaplasma: new challenges after reclassification. Rev. Sci. Technol. 34, 577-586. doi: $10.20506 /$ rst.34.2.2381

Lecuit, M., Sonnenburg, J. L., Cossart, P., and Gordon, J. I. (2007). Functional genomic studies of the intestinal response to a foodborne enteropathogen in a humanized gnotobiotic mouse model. J. Biol. Chem. 282, 15065-15072. doi: 10.1074/jbc.M610926200

Li, X. B., Gu, J. D., and Zhou, Q. H. (2015). Review of aerobic glycolysis and its key enzymes-new targets for lung cancer therapy. Thorac. Cancer 6, 17-24. doi: 10.1111/1759-7714.12148

Li, X., Jacobson, M. P., Zhu, K., Zhao, S., and Friesner, R. A. (2007). Assignment of polar states for protein amino acid residues using an interaction cluster decomposition algorithm and its application to high resolution protein structure modeling. Proteins 66, 824-837. doi: 10.1002/prot.21125

Madden, T. L., Tatusov, R. L., and Zhang, J. (1996). Applications of network BLAST server. Methods Enzymol. 266, 131-141. doi: 10.1016/S0076-6879(96)66011-X
Misra, R. M., Bajaj, M. S., and Kale, V. P. (2012). Vasculogenic mimicry of HT1080 tumour cells in vivo: critical role of HIF-1 $\alpha$-neuropilin-1 axis. PLoS ONE 7:e50153. doi: 10.1371/journal.pone.0050153

Munderloh, U. G., Jauron, S. D., Fingerle, V., Leitritz, L., Hayes, S. F., Hautman, J. M., et al. (1999). Invasion and intracellular development of the human granulocytic ehrlichiosis agent in tick cell culture. J. Clin. Microbiol. 37, 2518-2524

Pagé, E. L., Robitaille, G. A., Pouysségur, J., and Richard, D. E. (2002). Induction of hypoxia-inducible factor-1alpha by transcriptional and translational mechanisms. J. Biol. Chem. 277, 48403-48409. doi: 10.1074/jbc.M2091 14200

Pilot-Storck, F., Chopin, E., Rual, J. F., Baudot, A., Dobrokhotov, P., RobinsonRechavi, M., et al. (2010). Interactome mapping of the phosphatidylinositol 3 kinase-mammalian target of rapamycin pathway identifies deformed epidermal autoregulatory factor-1 as a new glycogen synthase kinase- 3 interactor. Mol. Cell. Proteomics 9, 1578-1593. doi: 10.1074/mcp.M900568-MCP200

Shin, J. H., Yang, J. Y., Jeon, B. Y., Yoon, Y. J., Cho, S. N., Kang, Y. H., et al. (2011). (1)H NMR-based metabolomic profiling in mice infected with Mycobacterium tuberculosis. J. Proteome Res. 10, 2238-2247. doi: 10.1021/pr1 $01054 \mathrm{~m}$

Somashekar, B. S., Amin, A. G., Rithner, C. D., Troudt, J., Basaraba, R., Izzo, A., et al. (2011). Metabolic profiling of lung granuloma in Mycobacterium tuberculosis infected guinea pigs: ex vivo $1 \mathrm{H}$ magic angle spinning NMR studies. J. Proteome Res. 10, 4186-4195. doi: 10.1021/pr2003352

Stuen, S., Granquist, E. G., and Silaghi, C. (2013). Anaplasma phagocytophiluma widespread multi-host pathogen with highly adaptive strategies. Front. Cell. Infect. Microbiol. 3:31. doi: 10.3389/fcimb.2013.00031

Sultana, H., Neelakanta, G., Kantor, F. S., Malawista, S. E., Fish, D., Montgomery, R. R., et al. (2010). Anaplasma phagocytophilum induces actin phosphorylation to selectively regulate gene transcription in Ixodes scapularis ticks. J. Exp. Med. 207, 1727-1743. doi: 10.1084/jem.20100276

Villar, M., Ayllón, N., Alberdi, P., Moreno, A., Moreno, M., Tobes, R., et al. (2015). Integrated metabolomics, transcriptomics and proteomics identifies metabolic pathways affected by Anaplasma phagocytophilum infection in tick cells. Mol. Cell. Proteomics 14, 3154-3172. doi: 10.1074/mcp.M115.0 51938

Wang, Q., Liang, B., Shirwany, N. A., and Zou, M. H. (2011). 2-Deoxy-D-glucose treatment of endothelial cells induces autophagy by reactive oxygen speciesmediated activation of the AMP-activated protein kinase. PLOS ONE 6:e17234. doi: 10.1371/journal.pone.0017234

Weidemann, A., Breyer, J., Rehm, M., Eckardt, K. U., Daniel, C., Cicha, I., et al. (2013). HIF-1 $\alpha$ activation results in actin cytoskeleton reorganization and modulation of Rac-1 signaling in endothelial cells. Cell. Commun. Signal. 11:80. doi: 10.1186/1478-811X-11-80

Zhou, J., Schmid, T., Frank, R., and Brüne, B. (2004). PI3K/Akt is required for heat shock proteins to protect hypoxia-inducible factor lalpha from pVHL-independent degradation. J. Biol. Chem. 279, 13506-13513. doi: 10.1074/jbc.M310164200

Ziello, J. E., Jovin, I. S., and Huang, Y. (2007). Hypoxia-Inducible Factor (HIF)-1 regulatory pathway and its potential for therapeutic intervention in malignancy and ischemia. Yale J. Biol. Med. 80, 51-60.

Conflict of Interest Statement: The authors declare that the research was conducted in the absence of any commercial or financial relationships that could be construed as a potential conflict of interest.

Copyright (c) 2017 Cabezas-Cruz, Alberdi, Valdés, Villar and de la Fuente. This is an open-access article distributed under the terms of the Creative Commons Attribution License (CC BY). The use, distribution or reproduction in other forums is permitted, provided the original author(s) or licensor are credited and that the original publication in this journal is cited, in accordance with accepted academic practice. No use, distribution or reproduction is permitted which does not comply with these terms. 